\title{
Pacific
}

Journal of

Mathematics

\section{AMENABLE CORRESPONDENCES AND APPROXIMATION PROPERTIES FOR VON NEUMANN ALGEBRAS}

\author{
Claire Anantharaman-Delaroche
}




\section{AMENABLE CORRESPONDENCES AND APPROXIMATION PROPERTIES FOR VON NEUMANN ALGEBRAS}

\section{Anantharaman-Delaroche}

We introduce the notion of amenable equivalence between von Neumann algebras, and study some approximation properties which remain invariant by this relation. We show for instance that the constant $\Lambda(M)$ associated with a von Neumann algebra $M$ when considering the weak* completely bounded approximation property is an invariant for this equivalence relation. As an example, let $\alpha$ be an amenable action of a locally compact group $G$ on a von Neumann algebra $M$; then the crossed product $M \rtimes G$ is amenably equivalent to $M$. Another example is obtained by considering a pair $G_{1} \subset G$ of locally compact groups such that the homogeneous space $G / G_{1}$ is amenable. Then the von Neumann algebras $W^{*}(G)$ and $W^{*}\left(G_{1}\right)$ generated by the left regular representations of $G$ and $G_{1}$ respectively are amenably equivalent. Therefore, if moreover $G$ is discrete, we get that $G$ and $G_{1}$ are simultaneously weakly amenable with the same Haagerup's constants $\Lambda_{G}=\Lambda_{G_{1}}$.

\section{Introduction}

Given a pair $M \subset N$ of von Neumann algebras, one finds many situations where there exists a norm one projection $E$ from $N$ onto $M$, and one may ask what properties of $N$ are automatically inherited by $M$ in this case, the most well-known example being the amenability of $N$. In this paper we will see that other approximation properties such as the weak* completely bounded approximation property $([\mathbf{H a a} 4],[\mathbf{C}-\mathbf{H}])$, or the $\sigma$-weak approximation property of $[\mathbf{K}]$ are also preserved. Their common feature is the approximation of the identity map of $N$ by appropriate $\sigma$-weakly continuous bounded maps, and the main problem is that the norm one projection $E$ is not $\sigma$-weakly continuous in general.

The existence of $E$ follows easily from the existence of a net $\left(\phi_{i}\right)_{i \in I}$ of $\sigma$-weakly continuous completely positive contractions $\phi_{i}: N \rightarrow M$ such that $\lim _{i} \phi_{i}(x)=x \sigma$-weakly for all $x \in M$. The converse is very likely true, but we are not able to prove it in full generality (see Cor. 3.9). At least we show that, when there exists a norm one projection $E$ from $N$ onto $M$, we may 
find a von Neumann algebra $N_{1}$, Morita equivalent to $N$, which contains $M$ as a von Neumann subalgebra, in such a way that there exists a net $\left(\phi_{i}\right)_{i \in I}$ of $\sigma$-weakly continuous completely positive contractions $\phi_{i}: N_{1} \rightarrow M$ with $\lim _{i} \phi_{i}(x)=x$ for all $x \in M$. This property is enough to prove the above results of heredity.

This latter relation between $M$ and $N$ is well expressed in terms of correspondences, and we work in this framework throughout this paper. Section 1 contains a discussion of the basic definitions concerning correspondences, as well as some notation. Let us only recall here that a correspondence from $M$ to $N$ is a Hilbert space $H$ with a pair of commuting normal representations $\pi_{M}$ and $\pi_{N^{0}}$ of $M$ and $N^{0}$ (the opposite of $N$ ) respectively, where the representations are always assumed to be faithful in this paper. We denote by $\mathcal{L}_{N^{0}}(H)$ the commutant of $\pi_{N^{0}}\left(N^{0}\right)$. Note that $\mathcal{L}_{N^{0}}(H)$ is Morita equivalent to $N$, and that we get in this way every von Neumann algebra Morita equivalent to $N$ [Rie2]. The notion of correspondence between two von Neumann algebras $M$ and $N$ was introduced by A. Connes ([Co5], [C-J]) and includes the concepts of group representations and completely positive maps as well. It is therefore a rich structure, which has proved to be useful in the study of a single von Neumann algebra, and in the comparison of two von Neumann algebras.

The connections between $M$ and $N$ we are interested in here are related to amenability. Recently, M. Bekka [Bek] has introduced a notion of amenability for an arbitrary unitary group representation which unifies several known notions in this field. In particular a unitary representation $\pi$ of a locally compact group $G$ is called amenable if the trivial representation of $G$ is weakly contained in $\pi \otimes \bar{\pi}$. In a similar way, we define in Section 2 a correspondence $H$ from $M$ to $N$ to be left amenable if the identity correspondence of $M$ is weakly contained in the correspondence $H \otimes_{N} \bar{H}$ from $M$ to $M$ (there is also an obvious definition for right amenability, which is not equivalent to left amenability). When $N=\mathbb{C}$, and $H=L^{2}(M)$ is the standard Hilbert space for $M$, left amenability means that the homomorphism $\eta$ from the algebraic tensor product $M \odot M^{\prime}$ into $\mathcal{L}(H)$, defined by $\eta\left(x \otimes x^{\prime}\right)=x x^{\prime}$ for $x \in M, x^{\prime} \in M^{\prime}$, has a bounded extension to the completion $M \otimes_{\min } M^{\prime}$. Thus $M$ is amenable in the terminology used in [Was]. More generally, let $N$ be a von Neumann subalgebra of $M$, and take $H=L^{2}(M)$, viewed as a correspondence from $M$ to $N$ (called the standard correspondence of the inclusion). Then the left amenability of $H$ is exactly the notion of amenability of $M$ relative to $N$ introduced and studied by S. Popa in [Po1]. Let us mention that S. Popa has recently introduced another notion of amenable inclusion [Po2], which is a key concept in the classification theory of subfactors, and is different from the one used in this paper. 
Let us recall also [E-L] that a von Neumann algebra $M$ is said to be semi-discrete if the identity map on $M$ can be approximated in the topology of simple weak* convergence by normal completely positive maps from $M$ to $M$ of finite rank, which preserve the identity. In [E-L], Effros and Lance proved that semi-discreteness is equivalent to amenability and that either property implies injectivity. In Section 2 of the present paper, these results are extended to the case of correspondences. We prove that a correspondence $H$ from $M$ to $N$ is left amenable if and only if the identity map of $M$ is the limit in the topology of simple weak* convergence of completely positive maps which admit factorizations through matrix algebras $M_{n}(N)$ with entries in $N$, as described in Theorem 2.2. We prove also that the left amenability of $H$ is equivalent to the existence of a net $\left(\phi_{i}\right)$ of completely positive maps from $N_{1}=\mathcal{L}_{N^{0}}(H)$ onto $M$, each of which has the form $x \mapsto \sum W_{j}^{*} x W_{j}$, where the sum is finite and the $W_{j}$ 's are bounded operators from $L^{2}(M)$ into $L^{2}\left(N_{1}\right)$ commuting with the obvious right actions of $M$. This in turn implies the existence of a norm one projection from $N_{1}$ onto $M$.

In Section 3, we define a correspondence $H$ from $M$ to $N$ to be left injective if there exists a norm one projection from $N_{1}=\mathcal{L}_{N^{0}}(H)$ onto $M$. Let us remark first that Bekka's definition is also related to ours in the following way. To every unitary representation $\pi$ of a locally compact group $G$ is canonically associated a correspondence $H_{\pi}$ from the von Neumann algebra $W^{*}(G)$ generated by the left regular representation, onto itself $[\mathbf{C - J}]$. We check (see Prop. 3.3) that if $\pi$ is amenable, then $H_{\pi}$ is left injective, and that the converse is true when $G$ is discrete.

Let $H$ be a left injective correspondence from $M$ to $N$. Following the ideas of [Co2] and [Was], we prove that when $M$ is semi-finite, or more generally when there is a normal faithful semi-finite weight on $M$ whose modular automorphism group is induced by unitaries in $\mathcal{L}_{N^{0}}(H)$, then $H$ is left amenable. When $N=\mathbb{C}$, this is Connes' result, and when $H$ is the standard correspondence of an inclusion $N \subset M$ of finite von Neumann algebras, this result has previously been obtained by Popa in [Po1].

Moreover, we prove that, given two von Neumann algebras $M$ and $N$, the existence of a left amenable correspondence from $M$ to $N$ is equivalent to the existence of a left injective correspondence from $M$ to $N$, that is to the existence of a von Neumann algebra $N_{1}$, Morita equivalent to $N$, containing $M$ in such a way that there is a norm one projection from $N_{1}$. onto $M$ (see Prop. 3.10). We say that $M$ is amenably dominated by $N$, and we write $M \prec N$, when such a property occurs. If moreover $N \prec M$, we say that $M$ and $N$ are amenably equivalent and we write $M \sim \underset{a}{\sim} N$. Let 
us consider for instance a $W^{*}$-dynamical system $(M, G, \alpha)$ where $\alpha$ is a continuous action of a locally compact group $G$ into $M$, and denote by $N$ the crossed product $M \rtimes G$. The correspondence $L^{2}(N)$ from $M$ to $N$ is left amenable (Prop. 2.6) and therefore there always exists a norm one projection from $N=\mathcal{L}_{N^{0}}\left(L^{2}(N)\right)$ onto $M$. Thus we have $M \prec M \rtimes G$. If moreover the dynamical system $(M, G, \alpha)$ is amenable [AD1], we have $\underset{a}{\sim} \underset{\alpha}{\sim} \underset{\alpha}{\sim} G$ (see Prop. 3.4). It follows in particular that every von Neumann algebra is amenably equivalent to a semi-finite von Neumann algebra. As another example, consider now a locally compact group $G$ and a closed subgroup $G_{1}$. Following Eymard [Eym], we say that the homogeneous space $G / G_{1}$ is amenable if there exists a $G$-invariant mean on $L^{\infty}\left(G / G_{1}\right)$. In this case, we show that $W^{*}(G) \underset{a}{\prec} W^{*}\left(G_{1}\right)$ and thus, obviously, $W^{*}(G) \underset{a}{\sim} W^{*}\left(G_{1}\right)$ when $G$ is moreover assumed to be discrete (see Prop. 3.5).

In many cases, the left injectivity of a correspondence from $M$ to $N$ is easier to check than the left amenability, but the approximation property arising from left amenability proves to be a very useful tool in Section 4, where we are interested in the study of properties which are invariant under amenable equivalence. Of course, we have to look for properties which are somewhat related to weak forms of amenability, the most interesting among them being the weak* completely bounded approximation property introduced by U. Haagerup [Haa4], $[\mathbf{C}-\mathbf{H}]$, which enables to distinguish the von Neumann algebras associated with lattices in simple Lie groups. Recall that a von Neumann algebra $M$ has the weak* completely boundedness approximation property if the identity map on $M$ can be approximated in the topology of simple weak* convergence by a net $\left(\phi_{i}\right)_{i \in I}$ of $\sigma$-weakly continuous finite rank operators such that for some $c \in \mathbb{R}^{+}$, and for all $i$, one has $\left\|\phi_{i}\right\|_{c b} \leq c$ (where \|\|$_{c b}$ is the completely bounded operator norm). The best constant $c$ for the existence of such a net is denoted by $\Lambda(M)$. When $M$ doesn't have this approximation property we put $\Lambda(M)=+\infty$. An analogous property is defined for a locally compact group $G$, as well as a constant $\Lambda_{G}$ (see [Haa4], [C-H]). When $M$ and $N$ are von Neumann algebras such that $M \underset{a}{\prec} N$, we prove (Th. 4.9) that $\Lambda(M) \leq \Lambda(N)$, and therefore $\Lambda(M)$ is an invariant for amenable equivalence. As a consequence, we get for instance that if $(M, G, \alpha)$ is a $W^{*}$-dynamical system, then $\Lambda(M) \leq \Lambda(M \underset{\alpha}{\rtimes} G)$, and that $\Lambda(M)=\Lambda(M \rtimes G)$ when the action is amenable. Also, given an exact sequence $0 \rightarrow G_{1}{ }^{\alpha} \rightarrow G \rightarrow G_{2} \rightarrow 0$ of discrete groups, where $G_{2}{ }^{\circ}$ is an amenable group, we have $\Lambda_{G_{1}}=\Lambda_{G}$. This result appears in contrast with Haagerup's result [Haa] showing that $\Lambda_{\left(\mathbb{Z}^{2} \rtimes S L(2 ; \mathbb{Z})\right)}=+\infty$, although $\Lambda_{\mathbb{Z}^{2}}=\Lambda_{S L(2 ; \mathbb{Z})}=1$. 


\section{Preliminaries}

For the reader's convenience, we begin by recalling the basic facts on correspondences that will be needed in this paper. For more details, the reader may consult $[\mathbf{C}-\mathbf{J}],[\mathbf{B D H}],[\mathbf{R i e 1}],[\mathbf{P o 1}]$, [Pas]. Let $M$ and $N$ be two von Neumann algebras.

1.1. A correspondence from $M$ to $N$ is a Hilbert space $H$ with a pair of commuting normal representations $\pi_{M}$ and $\pi_{N^{0}}$ of $M$ and $N^{0}$ (the opposite of $N)$ respectively $[\mathbf{C - J}]$. Usually, the triple $\left(H, \pi_{M}, \pi_{N^{0}}\right)$ will be denoted by $H$. For $x \in M, y \in N$ and $h \in H$, we shall write $x h y$ instead of $\pi_{M}(x) \pi_{N^{0}}(y) h$. In case of ambiguity on which algebras are acting, we shall write ${ }_{M} H_{N}$ instead of $H$. The commutants of $\pi_{M}(M)$ and $\pi_{N^{0}}\left(N^{0}\right)$ respectively will be denoted by $\mathcal{L}_{M}(H)$ and $\mathcal{L}_{N^{0}}(H)$. In this paper we shall always assume that $\pi_{M}$ and $\pi_{N^{0}}$ are faithful.

The standard form [Haa3] of $M$ gives rise to a correspondence $L^{2}(M)$ from $M$ to $M$, called the the identity correspondence, and denoted by $\operatorname{id}_{M}$. We will denote by $J_{M}$ the conjugate linear isometry of $L^{2}(M)$ given with the standard form of $M$. In this example, we have $\pi_{M^{0}}(x)=J_{M} x^{*} J_{M}$.

Let us recall first another useful equivalent way to look at correspondences. Let $X$ be a self-dual (right) Hilbert $N$-module (see [Pas]). The $N$-valued inner product, denoted by $\langle$,$\rangle , is supposed to be conjugate linear in the$ first variable and such that the linear span of $\{\langle\xi, \eta\rangle, \xi, \eta \in X\}$ is $\sigma$-weakly dense in $N$. The von Neumann algebra of all $N$-linear continuous operators from $X$ to $X$ will be denoted by $\mathcal{L}_{N}(X)$ (or $\mathcal{L}(X)$ when $N=\mathbb{C}$ ). Following ([BDH, Def. 2.1]), by a $M-N$ correspondence we mean a pair $(X, \pi)$ where $X$ is as above, and $\pi$ is a unital normal faithful homomorphism from $M$ into $\mathcal{L}_{N}(X)$. More briefly, such a correspondence will be denoted by $X$ and we shall often write $x \xi$ instead of $\pi(x) \xi$.

These two notions of correspondences are related in the following way. Consider a $M-N$ correspondence $X$ and let $H(X)=X \otimes_{N} L^{2}(N)$ be the Hilbert space obtained by inducing the standard representation of $N$ up to $M$ via $X$ ([Rie1, Th. 5.1]). Then the left action of $M$ and the right action of $N$ defined on $H(X)$ by

$$
x(\xi \otimes h) y=x \xi \otimes h y, \quad \text { for } \xi \in X, h \in L^{2}(N), x \in M, y \in N,
$$

turn $H(X)$ into a correspondence between these algebras.

Conversely, given a correspondence $H$ between $M$ and $N$, let $X(H)$ bethe space $\operatorname{Hom}_{N^{0}}\left(L^{2}(N), H\right)$ of continuous $N^{0}$-linear operators from $L^{2}(N)$ into $H$. Let $N$ acts on the right of $X(H)$ by composition of operators and define on $X(H)$ a $N$-valued inner product by $\langle r, s\rangle=r^{*} s$ for $r, s \in X(H)$. Then $X(H)$ is a self-dual Hilbert $N$-module ([Rie1, Th. 6.5]). Moreover, 
$M$ acts on the left of $X(H)$ by composition of operators, and we obtain in this way a $M-N$ correspondence.

The maps $X \mapsto H(X)$ and $H \mapsto X(H)$ are inverse to each other ([BDH, Th. 2.2] and [Rie1, Prop. 6.10]), up to unitary equivalence. We shall not make any distinction between equivalent correspondences. Also, we shall often identify a correspondence $H$ and its self-dual module version $X(H)$. It will be useful to note that, when replacing $X$ by $H=H(X)=$ $X \otimes_{N} L^{2}(N)$, the von Neumann algebra $\mathcal{L}_{N}(X)$ is canonically identified with $\mathcal{L}_{N^{0}}(H)$, by the map which carries $x \in \mathcal{L}_{N}(X)$ onto the element (still denoted by $x$ ) of $\mathcal{L}_{N^{0}}(H)$ defined as follows :

$$
x(\xi \otimes h)=(x \xi) \otimes h, \quad \text { for } \xi \in X, h \in L^{2}(N) .
$$

Let us recall from [Rie1] that two von Neumann $M$ and $N$ are Morita equivalent if there exists a $M-N$ correspondence $X$ (or equivalently a correspondence $H$ from $M$ to $N$ ) such that $M$ is isomorphic to $\mathcal{L}_{N}(X)$ (or $\mathcal{L}_{N^{0}}(H)$ ). This amounts to saying that there is a type $I$ factor $F$ and a projection $e$ in $F \otimes N$ with central support 1 such that $M$ and the reduced von Neumann algebra $e(F \otimes N) e$ are isomorphic.

Let us point out now that most of the familiar techniques used in von Neumann algebras theory and Hilbert spaces theory apply also when we work with a self-dual Hilbert $N$-module $X$. The ultrastrong topology has a useful analogue on $X$ called the s-topology (see [BDH, §1.3]). It is the topology defined on $X$ by the family of semi-norms $q_{\varphi}$, where $\varphi$ is a normal positive form on $N$ and

$$
q_{\varphi}(\eta)=\varphi(\langle\eta, \eta\rangle)^{1 / 2}, \quad \text { for } \eta \in X .
$$

1.2. The usual notions in the theory of representations of groups have analogues in the theory of correspondences, that we recall briefly now.

Let $H$ be a correspondence from $M$ to $N$, and let $\bar{H}$ be the conjugate Hilbert space. If $h \in H$, we denote by $\bar{h}$ the vector $h$ when viewed as an element of $\bar{H}$. Then $\bar{H}$ has a natural structure of correspondence from $N$ to $M$ by

$$
y \bar{h} x=\overline{x^{*} h y^{*}}, \quad \text { for } x \in M, y \in N, h \in H
$$

(see [Po1, 1.3.7]). It is called the adjoint or conjugate correspondence of $H$. Notice also that with a $M-N$ correspondence $X=X(H)$ is associated its adjoint $\bar{X}=X(\bar{H})$. But in general, there is no explicit description of $\bar{X}$ from $X$.

A subcorrespondence of $H$ is a Hilbert subspace $K$ of $H$, stable by the left $M$-action and the right $N$-action. In the self-dual version, a subcorrespondence $Y$ of $X$ is a submodule of $X$ closed in the $s$-topology and stable by 
the left action of $M$. In this case, we shall say that $K$ is contained in $H$ (or that $Y$ is contained in $X$ ), and we shall write $K \leq H$ (or $Y \leq X$ ).

Consider now three von Neumann algebras $M, N, P$, a $M-N$ correspondence $(X, \pi)$ and a $N-P$ correspondence $\left(Y, \pi_{1}\right)$. We denote by $X \otimes_{N} Y$ the self-dual completion of the algebraic tensor product $X \odot Y$ endowed with the obvious right action of $P$ and the $P$-valued inner product

$$
\left\langle\xi \otimes \eta, \xi_{1} \otimes \eta_{1}\right\rangle_{P}=\left\langle\eta,\left\langle\xi, \xi_{1}\right\rangle_{N} \eta_{1}>_{P}, \quad \text { for } \xi, \xi_{1} \in X, \eta, \eta_{1} \in Y .\right.
$$

Then there is a canonical homomorphism from $\mathcal{L}_{N}(X)$ into $\mathcal{L}_{P}\left(X \otimes_{N} Y\right)$, sending $x \in \mathcal{L}_{N}(X)$ to the map $\xi \otimes \eta \mapsto(x \xi) \otimes \eta$. Moreover this homomorphism is faithful when $\pi_{1}: N \rightarrow \mathcal{L}_{P}(Y)$ is faithful ([AD3, Lemma 1.5]). By composition of this homomorphism with $\pi$, we get a left action of $M$ into $X \otimes_{N} Y$ which turns $X \otimes_{N} Y$ into a $M-P$ correspondence, called the composition correspondence of $X$ by $Y$. Put $H=H(X)$ and $K=H(Y)$. When an auxiliary faithful weight $\nu$ has been chosen on $N$, A. Connes has shown [C] how to define the composition $H \otimes_{\nu} K$ of the correspondences $H$ and $K$ (see also [S]). It follows from ([S, Prop. 2.6]) that, up to equivalence, the result does not depend on the choice of $\nu$, so we shall use the notation $H \otimes_{N} K$ instead of $H \otimes_{\nu} K$. It is easily checked that $X\left(H \otimes_{N} K\right)=X \otimes_{N} Y$ and thus there is no ambiguity on the notion of composition of correspondences.

1.3. Let $N$ be a von Neumann subalgebra of a von Neumann algebra $M$ (and then we will say that $N \subset M$ is a pair of von Neumann algebras). The Hilbert space $L^{2}(M)$ has a natural structure of correspondence from $M$ to $N$ by restricting to $N$ the right action of $M$. This object, which is crucial in the study of the inclusion, is called the standard correspondence associated with the pair $N \subset M$ and denoted by ${ }_{M} L^{2}(M)_{N}$. In a similar way, we may consider the correspondence ${ }_{N} L^{2}(M)_{M}$ from $N$ to $M$, and by using $J_{M}$ it is easily checked that it is equivalent to the conjugate of ${ }_{M} L^{2}(M)_{N}$. Let us remark that any correspondence ${ }_{M} H_{N}$ is isomorphic to the correspondence ${ }_{M} L^{2}(M) \otimes_{M} H_{N}$, and to ${ }_{M} H \otimes_{N} L^{2}(N)_{N}$ as well.

Let $H$ be a correspondence from $M$ to $M$. Then ${ }_{N} L^{2}(M) \otimes_{M} H \otimes_{M}$ $L^{2}(M)_{N}$ is the correspondence from $N$ to $N$ obtained by restricting to $N$ the left and right actions of $M$ on $H$. It will be called the restriction of $H$ from $M$ to $N$.

Let $H$ be now a correspondence from $N$ to $N$. Then ${ }_{M} L^{2}(M) \otimes_{N} H \otimes_{N}$ $L^{2}(M)_{M}$ is a correspondence from $M$ to $M$. We will say that it is the correspondence induced by $H$ from $N$ up to $M$ and will denote it by $\operatorname{Ind}_{N}^{M} H$.

1.4. Let us recall now that a correspondence $H$ from a von Neumann algebra $M$ to a von Neumann algebra $N$ is nothing else than a representation of the binormal tensor product $M \otimes_{\mathrm{bin}} N^{0}$ (see [E-L] for the definition 
of the norm bin). Furthermore two correspondences are isomorphic if and only if they are unitarily equivalent when considered as representations of $M \otimes_{\text {bin }} N^{0}$. Thus every notion which makes sense for representations of $C^{*}$ algebras can also be defined for correspondences. In particular the topology defined by Fell ([Fel, Section 1]) on the space of (equivalence classes of) representations of the $C^{*}$-algebra $M \otimes_{\text {bin }} N^{0}$ gives rise to the following topology on the set $\operatorname{Corr}(M, N)$ of (equivalence classes of) correspondences from $M$ to $N$ (as usual this set is restricted suitably in order to avoid paradoxically huge sets). Let $H_{0} \in \operatorname{Corr}(M, N), \varepsilon>0, E \subset M$ and $F \subset N$ two finite sets, and $S=\left\{h_{1}, \ldots, h_{p}\right\}$ a finite subset of $H_{0}$. We define by $U\left(H_{0} ; \varepsilon, E, F, S\right)$ the set of $H \in \operatorname{Corr}(M, N)$ such that there exist $k_{1}, \ldots, k_{p} \in H$ with

$$
\left|\left\langle k_{i}, x k_{j} y\right\rangle-\left\langle h_{i}, x h_{j} y\right\rangle\right|<\varepsilon \quad \text { for all } x \in E, y \in F, i, j=1, \ldots, p .
$$

Then $\operatorname{Corr}(M, N)$ is equipped with the well defined topology having these $U$ 's as a basis of neighbourhoods.

If we regard correspondences as self-dual Hilbert modules, the topology may be described as follows (see [AD3, §1.12]). Let $X_{0}=X\left(H_{0}\right), \mathcal{V}$ a $\sigma$ weak neighbourhood of 0 in $N, E$ a finite subset of $M$, and $S=\left\{\xi_{1}, \ldots, \xi_{p}\right\}$ a finite subset of $X_{0}$ be given. We denote by $V\left(X_{0} ; \mathcal{V}, E, S\right)$ (or, more briefly $V(\mathcal{V}, E, S))$ the set of correspondences $X$ such that there exist $\eta_{1}, \ldots, \eta_{p} \in X$ with

$$
\left\langle\eta_{i}, x \eta_{j}\right\rangle-\left\langle\xi_{i}, x \xi_{j}\right\rangle \in \mathcal{V} \quad \text { for all } x \in E, i, j=1, \ldots, p .
$$

Then such $V$ 's constitute a basis of neighbourhoods of $X_{0}$ in $\operatorname{Corr}(M, N)$. Moreover, if $X_{0}$ has a cyclic vector $\xi_{0}$, it has a basis of neighbourhoods of the form $V\left(X_{0} ; \mathcal{V}, E\right)=V\left(X_{0} ; \mathcal{V}, E,\left\{\xi_{0}\right\}\right)$. Note that in this case, $X_{0}$ belongs to the closure of $X \in \operatorname{Corr}(M, N)$ if and only if there is a net $\left(\xi_{i}\right)$ in $X$ such that $\lim _{i}\left\langle\xi_{i}, x \xi_{i}\right\rangle=\left\langle\xi_{0}, x \xi_{0}\right\rangle \sigma$-weakly for all $x \in M$. If $\xi \in X$, we say that the normal completely positive map $x \mapsto\langle\xi, x \xi\rangle$ from $M$ to $N$ is a coefficient of $X$.

In particular, the identity correspondence of $M$ belongs to the closure of a $M-M$ correspondence $X$ if and only if there is a net $\left(\phi_{i}\right)$ of coefficients of $X$ such that $\lim _{i} \phi_{i}(x)=x \sigma$-weakly for all $x \in M$.

1.5. Let $H_{0}, H_{1}$ be two correspondences from $M$ to $N$, and denote by $\pi_{0}$ and $\pi_{1}$ the associated representations of $M \otimes_{\mathrm{bin}} N^{0}$. We say that $H_{0}$ is weakly contained in $H_{1}$, and we write $H_{0} \prec H_{1}$ if the representation $\pi_{0}$ is weakly contained in $\pi_{1}$, that is if $\operatorname{Ker} \pi_{0} \supset \operatorname{Ker} \pi_{1}$. This amounts to saying that $H_{0}$ belongs to the closure of the set of finite direct sums of copies of $H_{1}$ in $\operatorname{Corr}(M, N)$ (see [Fel, Th. 1.1]). For instance, the identity correspondence of $M$ is weakly contained in a $M-M$ correspondence $X$ if and only if there exists a net $\left(\phi_{i}\right)$ of completely positive maps from $M$ to $M$, each of which 
is a finite sum of coefficients of $X$, such that $\lim _{i} \phi_{i}(x)=x$ for all $x \in M$. Note that we may replace the net $\left(\phi_{i}\right)$ by a bounded one, thanks to Lemma 2.2 of $[\mathrm{AD} 4]$ that we recall now.

Lemma 1.6. Let $C P(M, N)$ denote the set of completely positive maps from a von Neumann algebra $M$ into a von Neumann algebra $N$, equipped with the topology of pointwise $\sigma$-weak convergence. Let $F$ be a convex subcone of $C P(M, N)$ such that for $\phi \in F$ and $b \in N$, the completely positive map $x \mapsto b^{*} \phi(x) b$ belongs to $F$. Let $\phi$ be an element of the closure of $F$ in $C P(M, N)$. Then there exists a net $\left(\phi_{\imath}\right)$ in $F$ such that $\phi_{\imath}(1) \leq \phi(1)$ for all $i$, which converges to $\phi$.

Lemma 1.7. Let $M, N, P$ be von Neumann algebras, and let $H, K$ be two correspondences from $M$ to $N$ with $H \prec K$.

a) For every correspondence $L$ from $P$ to $M$, we have $L \otimes_{M} H \prec L \otimes_{M} K$.

b) For every correspondence $L$ from $N$ to $P$, we have $H \otimes_{N} L \prec K \otimes_{N} L$.

Proof. a) We will show that in every neighbourhood $V(\mathcal{V}, E, S)$ of $X(L) \otimes_{M}$ $X(H)$, there is a finite multiple of $X(L) \otimes_{M} X(K)$, where $\mathcal{V}$ is a $\sigma$-weak neighbourhood of 0 in $N, E$ a finite subset of $P$, and $S=\left\{\eta_{1}, \ldots, \eta_{p}\right\}$ a finite subset of $X(L) \otimes_{M} X(K)$. We may suppose that each $\eta_{i}$ is in the algebraic tensor product $X(L) \odot X(H)$, that is $\eta_{i}=\sum_{k} \xi_{k}^{i} \otimes \zeta_{k}^{i}$ with $\xi_{k}^{i} \in X(L)$ and $\zeta_{k}^{i} \in X(H)$. We have then

$$
\begin{aligned}
\left\langle\eta_{i}, x \eta_{j}\right\rangle & =\sum_{k, l}\left\langle\xi_{k}^{i} \otimes \zeta_{k}^{2}, x \xi_{l}^{\jmath} \otimes \zeta_{l}^{\jmath}\right\rangle \\
& =\sum_{k, l}\left\langle\zeta_{k}^{i},\left\langle\xi_{k}^{i}, x \xi_{l}^{j}\right\rangle \zeta_{l}^{j}\right\rangle .
\end{aligned}
$$

Choose a $\sigma$-weak neighbourhood $\mathcal{W}$ of 0 in $N$ and consider the neighbourhood $V\left(\mathcal{W}, E^{\prime}, S^{\prime}\right)$ of $X(H)$ where $S^{\prime}$ is the finite subset of $X(H)$ formed by all the $\zeta_{k}^{i}$,s, and $E^{\prime}$ is the finite subset of $M$ formed by the $\left\langle\xi_{k}^{2}, x \xi_{l}^{\jmath}\right\rangle$ for all $i, j, k, l$ and $x \in E$. By hypothesis, this neighbourhood contains a finite multiple $Y$ of $X(K)$, that is we may find vectors $\tau_{k}^{i}$ in $Y$ such that, in particular

$$
\left\langle\zeta_{k}^{i},\left\langle\xi_{k}^{i}, x \xi_{l}^{j}\right\rangle \zeta_{l}^{j}\right\rangle-\left\langle\tau_{k}^{i},\left\langle\xi_{k}^{i}, x \xi_{l}^{j}\right\rangle \tau_{l}^{j}\right\rangle \in \mathcal{W}
$$

for all $i, j, k, l$, and $x \in E$. Put $\eta_{i}^{\prime}=\sum_{k} \xi_{k}^{i} \otimes \tau_{k}^{i}$. Then $\eta_{i}^{\prime}$ belongs to $X(L) \otimes_{M} Y$ which is a finite multiple of $X(L) \otimes_{M} X(K)$, and obviously, if $\mathcal{W}$ is small enough, we have

$$
\left\langle\eta_{i}, x \eta_{j}\right\rangle-\left\langle\eta_{i}^{\prime}, x \eta_{j}^{\prime}\right\rangle \in \mathcal{V} \quad \text { for all } i, j \text { and } x \in E .
$$

In a similar way, we may prove b). 
Corollary 1.8. Let $N \subset M$ be a pair of von Neumann algebras, and $H, K$ two correspondences from $N$ to $N$ with $H \prec K$. Then the induced correspondence $\operatorname{Ind}_{N}^{M} H$ from $M$ to $M$ is weakly contained in $\operatorname{Ind}_{N}^{M} K$.

\section{Amenable and semi-discrete correspondences}

Definition 2.1. We say that a correspondence $H$ from $M$ to $N$ is left amenable if $\operatorname{id}_{M} \prec H \otimes_{N} \bar{H}$. We say that $H$ is right amenable if $\bar{H}$ is left amenable, that is if id $\operatorname{id}_{N} \prec \bar{H} \otimes_{M} H$.

Put $N_{1}=\mathcal{L}_{N^{0}}(H)$. Sauvageot has proved ([S, Prop. 3.1]) that $H \otimes_{N} \bar{H}$ is a standard form for $N_{1}$, and thus we have

$$
{ }_{M} H \otimes_{N} \bar{H}_{M}={ }_{M} L^{2}\left(N_{1}\right)_{M}={ }_{M} L^{2}\left(N_{1}\right) \otimes_{N_{1}} L^{2}\left(N_{1}\right)_{M} .
$$

Therefore we see that ${ }_{M} H_{N}$ is left amenable if and only if ${ }_{M} L^{2}\left(N_{1}\right)_{N_{1}}$ is left amenable, and thus it would be enough to study the case of an inclusion $M \subset N_{1}$ with $H={ }_{M} L^{2}\left(N_{1}\right)_{N_{1}}$.

Following ([Po1, Def. 3.2]), we say that a von Neumann algebra $M$ is amenable relative to a von Neumann subalgebra $N$ if the standard correspondence ${ }_{M} L^{2}(M)_{N}$ of the inclusion is left amenable. Let us remark that for a pair $N \subset M$ of type $\mathrm{II}_{1}$ factors, ${ }_{M} L^{2}(M)_{N}$ is right amenable, since id ${ }_{N}$ is contained in ${ }_{N} L^{2}(M)_{N}$, although ${ }_{M} L^{2}(M)_{N}$ is not always left amenable.

Theorem 2.2. Let $H$ be a correspondence from $M$ to $N$. Then the following properties are equivalent :

i) $H$ is left amenable;

ii) there exists a net of completely positive maps $\phi_{i}: M \rightarrow M$, such that $\phi_{i}(x)$ converges $\sigma$-weakly to $x$ for all $x \in M$, where each $\phi_{i}$ is a finite sum of composed maps $\psi \circ \theta: M \rightarrow M_{n}(\mathbb{C}) \otimes N \rightarrow M$ of the following type: there is an integer $n$, elements $T_{i} \in \operatorname{Hom}_{N^{0}}\left(L^{2}(N), H\right)$ and $S_{i} \in \operatorname{Hom}_{M}\left(L^{2}(M), H\right), 1 \leq i \leq n$, such that

$$
\begin{aligned}
& \theta(x)=\left(\begin{array}{c}
T_{1}^{*} \\
\vdots \\
T_{n}^{*}
\end{array}\right) x\left(T_{1}, \ldots, T_{n}\right)=\left(T_{i}^{*} x T_{j}\right) \in M_{n}(\mathbb{C}) \otimes N \quad \text { for all } x \in M, \\
& \psi(x)=\left(J_{M} S_{1}^{*}, \ldots, J_{M} S_{n}^{*}\right)\left(\pi_{N^{0}}\left(x_{i j}^{*}\right)\right)\left(\begin{array}{c}
S_{1} J_{M} \\
\vdots \\
S_{n} J_{M}
\end{array}\right) \in M,
\end{aligned}
$$

for all $x=\left(x_{i j}\right) \in M_{n}(\mathbb{C}) \otimes N$. 
Proof. Put $X=X(H)=\operatorname{Hom}_{N^{0}}\left(L^{2}(N), H\right)$. Then, as it has been recalled in 1.5 above, $\operatorname{id}_{M}$ is weakly contained in $X \otimes_{N} \bar{X}$ if and only if the identity map of $M$ is the limit in the topology of pointwise $\sigma$-weak convergence of a net of completely positive maps of the form $x \mapsto \sum_{i}\left\langle\xi_{i}, x \xi_{i}\right\rangle$ where the sum is finite, and the $\xi_{i}$ 's are in $X \otimes_{N} \bar{X}$. Thanks to Lemma 1.6 we may suppose that $\sum_{i}\left\langle\xi_{i}, \xi_{i}\right\rangle \leq 1$. Furthermore, by the Kaplansky density theorem, given $\varphi_{1}, \ldots, \varphi_{p}$ in $M_{*}^{+}, \varepsilon>0$, and $\xi \in X \otimes_{N} \bar{X}$, we may find $\eta$ in $X \odot \bar{X}$ with $\|\eta\| \leq\|\xi\|$ and $q_{\varphi_{z}}(\xi-\eta) \leq \varepsilon$ for $i=1, \ldots, p$. Then for $x \in M$ we have

$$
\begin{aligned}
\left|\varphi_{i}(\langle\xi, x \xi\rangle)-\varphi_{i}(\langle\eta, x \eta\rangle)\right| & \leq\left|\varphi_{i}(\langle\xi-\eta, x \xi\rangle)\right|+\left|\varphi_{i}(\eta, x(\xi-\eta))\right| \\
& \leq\left[\varphi_{i}(\langle x \xi, x \xi\rangle)\right]^{1 / 2} q_{\varphi_{i}}(\xi-\eta) \\
& \quad+\left[\varphi_{i}(\langle\eta, \eta\rangle)\right]^{1 / 2} q_{\varphi_{i}}(x(\xi-\eta)) \\
& \leq 2 \varepsilon\|x\|\left\|\varphi_{i}\right\|^{1 / 2}\|\xi\| .
\end{aligned}
$$

Thus, it is easily seen that we may take the $\xi_{i}$ 's above in $X \odot \bar{X}$.

Now, we consider $\eta=\sum_{k} T_{k} \otimes R_{k} \in X \odot \bar{X}$, with $T_{k} \in X=\operatorname{Hom}_{N^{0}}\left(L^{2}(N)\right.$, $H)$, and $R_{k} \in \bar{X}=\operatorname{Hom}_{M^{0}}\left(L^{2}(M), \bar{H}\right)$. We have

$$
\langle\eta, x \eta\rangle=\sum_{k, l}\left\langle T_{k} \otimes R_{k}, x T_{l} \otimes R_{l}\right\rangle=\sum_{k, l} R_{k}^{*}\left(T_{k}^{*} x T_{l}\right) R_{l} .
$$

Denote by $j: \bar{H} \rightarrow H$ the canonical antilinear isomorphism and put $S_{k}=$ $j R_{k} J_{M}$. Then $S_{k} \in \operatorname{Hom}_{M}\left(L^{2}(M), H\right)$, and for $y \in N$, acting on $\bar{H}$ by $j \pi_{N^{0}}(y)^{*} j$, we have

$$
R_{k}^{*} y R_{l}=J_{M} S_{k}^{*} j y j S_{l} J_{M}=J_{M} S_{k}^{*} \pi_{N^{0}}\left(y^{*}\right) S_{l} J_{M} .
$$

The proof of the theorem follows at once.

2.3. Let us apply Theorem 2.2 in the case where $M \subset N$ is a pair of von Neumann algebras and $H={ }_{M} L^{2}(N)_{N}$. Then $T_{k} \in \operatorname{Hom}_{N^{0}}\left(L^{2}(N), L^{2}(N)\right)=$ $N$. If we put $R_{k}=J_{N} S_{k} J_{M} \in \operatorname{Hom}_{M^{0}}\left(L^{2}(M), L^{2}(N)\right)$, we have, for $x \in M$,

$$
\begin{aligned}
\sum_{k, l} J_{M} S_{k}^{*} \pi_{N^{0}}\left(T_{l}^{*} x^{*} T_{k}\right) S_{l} J_{M} & =\sum_{k, l} R_{k}^{*} T_{k}^{*} x T_{l} R_{l} \\
& =W^{*} x W
\end{aligned}
$$

with $W=\sum_{k} T_{k} R_{k} \in \operatorname{Hom}_{M^{0}}\left(L^{2}(M), L^{2}(N)\right)$.

Thus, we get the (less precise but useful) statement that ${ }_{M} L^{2}(N)_{N}$ is left amenable if and only if there exists a net of finite sequences $\left(W_{1}^{i}, \ldots, W_{p_{i}}^{i}\right)_{\imath \in I}$ of elements of $\operatorname{Hom}_{M^{0}}\left(L^{2}(M), L^{2}(N)\right)$ such that, for $x \in M$, we have

$$
\lim _{i} \sum_{1 \leq j \leq p_{\imath}}\left(W_{j}^{i}\right)^{*} x W_{j}^{i}=x
$$


$\sigma$-weakly (this could have been easily seen directly since ${ }_{M} L^{2}(N){ }_{M}={ }_{M} L^{2}(N)$ $\left.\otimes_{N}{\overline{L^{2}(N)}}_{M}\right)$.

Proposition 2.4. Let $H$ be a correspondence from $M$ to $N$ and put $N_{1}=$ $\mathcal{L}_{N^{0}}(H)$. Then the following conditions are equivalent :

i) $H$ is left amenable;

ii) there exists a net $\left(\phi_{i}\right)$ of completely positive maps from $N_{1}$ to $M$, of the form $\phi_{i}(x)=\sum_{j}\left(W_{j}^{i}\right)^{*} x W_{j}^{i}$, where the sum is finite and $W_{j}^{i} \in$ $\operatorname{Hom}_{M^{0}}\left(L^{2}(M), L^{2}\left(N_{1}\right)\right)$, such that $\lim _{i} \phi_{i}(x)=x \sigma$-weakly for all $x \in$ $M$.

Proof. This corollary follows immediately from the observations made in 2.1 and 2.3.

Proposition 2.5. Let $H$ be a correspondence from $M$ to $N$ and put $N_{1}=$ $\mathcal{L}_{N^{0}}(H)$. Consider the following conditions.

i) $H$ is left amenable;

ii) there exists a net $\left(\phi_{i}\right)$ of normal completely positive maps from $N_{1}$ to $M$, such that $\phi_{i}(1) \leq 1$ for all $i$ and $\lim _{i} \phi_{i}(x)=x \sigma$-weakly for all $x \in M$;

iii) there exists a norm one projection from $N_{1}$ onto $M$.

Then i) $\Rightarrow$ ii) $\Rightarrow$ iii).

Proof. i) $\Rightarrow$ ii) is a consequence of Proposition 2.4 and Lemma 1.6.

ii) $\Rightarrow$ iii) Denote by $\mathcal{L}\left(N_{1}, M\right)$ the space of all bounded maps from $N_{1}$ into $M$. This space is canonically identified to the dual of the projective tensor product $N_{1} \hat{\otimes} N_{*}$, and on the unit ball of $\mathcal{L}\left(N_{1}, M\right)$, the weak* topology coincides with the topology of pointwise $\sigma$-weak convergence. Then it is easily checked that any accumulation point of the net $\left(\phi_{i}\right)$ is a norm one projection from $N_{1}$ onto $M$.

By a dynamical system $(M, G, \alpha)$, we mean a continuous action of a locally compact group $G$ on a von Neumann algebra $M$. In other words, $\alpha$ is an homomorphism from $G$ into the group Aut $M$ of all automorphisms of $M$, with $g \mapsto \alpha_{g}(x) \sigma$-weakly continuous for all $x \in M$. Then we have the following example of left amenable correspondence.

Proposition 2.6. Let $(M, G, \alpha)$ be a dynamical system and denote by $N$ the crossed product $M_{\alpha} G$. Then ${ }_{M} L^{2}(N)_{N}$ is a left amenable correspondence, and therefore there exists a norm one projection from $M_{\alpha}^{\rtimes} G$ onto $M$.

Proof. The left and right actions of $M$ on $L^{2}(M)$ will be denoted by $\pi_{M}$ and $\pi_{M^{0}}$ respectively, and $\rho_{M}$ and $\rho_{M^{0}}$ will denote the left and right actions of 
$M$ on $L^{2}(N)$. We will prove that, given $x_{1}, \ldots, x_{p}, y_{1}, \ldots, y_{p} \in M$, we have

$$
\left\|\sum_{i} \pi_{M}\left(x_{i}\right) \pi_{M^{0}}\left(y_{i}\right)\right\| \leq\left\|\sum_{i} \rho_{M}\left(x_{i}\right) \rho_{M^{0}}\left(y_{i}\right)\right\| .
$$

But $L^{2}(M) \otimes L^{2}(G)$ is a standard form for $N$, and for $x, y \in M$ and $\xi \in$ $L^{2}(M) \otimes L^{2}(G)$ we have

$$
\left(\rho_{M}(x) \rho_{M^{0}}(y) \xi\right)(s)=\left(\pi_{M}\left(\alpha_{s^{-1}}(x)\right) \pi_{M^{0}}(y) \xi\right)(s) .
$$

Thus $\sum_{i} \rho_{M}\left(x_{\imath}\right) \rho_{M^{0}}\left(y_{i}\right)$ is a decomposable operator, and since

$$
s \mapsto\left\|\sum_{i} \pi_{M}\left(\alpha_{s^{-1}}\left(x_{i}\right)\right) \pi_{M^{0}}\left(y_{i}\right)\right\|
$$

is lower semi-continuous, we get (1).

2.7. Let us examine now the statement of Theorem 2.2 when $N \subset M$ is a pair of von Neumann algebras and $H={ }_{M} L^{2}(M)_{N}$. In this case, we have

$$
S_{\imath} \in \operatorname{Hom}_{M}\left(L^{2}(M), L^{2}(M)\right)=J_{M} M J_{M}
$$

and we set $R_{k}=J_{M} S_{k} J_{M}$. Then, for $x \in M$, we get

$$
\sum_{k, l} J_{M} S_{k}^{*} \pi_{N^{0}}\left(T_{l}^{*} x^{*} T_{k}\right) S_{l} J_{M}=\sum_{k, l} R_{k}^{*}\left(T_{k}^{*} x T_{l}\right) R_{l}
$$

Moreover, if we take $N=\mathbb{C}$, each $T_{k}$ is given by a vector $\xi_{k}$ in $L^{2}(M)$, and $T_{k}^{*} x T_{l}=\left\langle\xi_{k}, x \xi_{l}\right\rangle$. Therefore we see that $L^{2}(M)$ is a left amenable correspondence from $M$ to $\mathbb{C}$ if and only if the identity map of $M$ is the $\sigma$-weak pointwise limit of completely positive maps which are finite sums of maps of the form $x \mapsto \sum_{k, l}\left\langle\xi_{k}, x \xi_{l}\right\rangle m_{k}^{*} m_{l}$, with $m_{k} \in M$ and $\xi_{k} \in L^{2}(M)$ for all $k$. This implies the existence of approximate factorizations through matrix algebras for the identity map of $M$, and in fact, using density arguments as in ([AD4, Lemma 4.3]), we easily see that the two properties are equivalent.

This motivates the following definition.

Definition 2.8. We say that a correspondence $H$ from $M$ to $N$ is left semidiscrete if the identity map of $M$ is the pointwise $\sigma$-weak limit of finite sums of maps of the form $\psi \circ \theta$, with $\theta: M \rightarrow M_{n}(\mathbb{C}) \otimes N, \quad \psi: M_{n}(\mathbb{C}) \otimes N \rightarrow M$ as in the statement of Theorem 2.2. If $N \subset M$ is a pair of von Neumann algebras, and $H={ }_{M} L^{2}(M)_{N}$, we shall also say that $M$ is semi-discrete relative to $N$. 
2.9. Let us consider again a pair $N \subset M$ of von Neumann algebras and $H=$ ${ }_{M} L^{2}(M)_{N}$, and let us suppose moreover the existence of a faithful normal conditional expectation $E$ from $M$ onto $N$. Then $X=X(H)$ is the self-dual completion of $M$ endowed with the obvious structures of left $M$-module and right $N$-module, and the $N$-valued inner product $\left\langle m, m_{1}\right\rangle=E\left(m^{*} m_{1}\right)$ (see [AD3, §1.8]), and $\bar{X}=M$ viewed as a self-dual right $M$-module and left $N$-module. Using the density of $M \odot M$ into $X \otimes_{N} \bar{X}$ in the $s$-topology, and standard approximation arguments as in the proof of Theorem 2.2, we see that ${ }_{M} H_{N}$ is left amenable if and only if the identity map of $M$ is the $\sigma$-weak pointwise limit of completely positive maps which are finite sums of maps of the form $x \in M \mapsto \sum_{i, j \in J} m_{i}^{*} E\left(a_{i}^{*} x a_{j}\right) m_{j}$, with $J$ finite and $a_{i}, m_{i} \in M$ for $i \in J$.

In [Po1], S. Popa posed the question of finding a formulation of the relative amenability of a pair $N \subset M$ of $\mathrm{II}_{1}$ factors in terms of the existence of nets of appropriate normal completely positive maps from $M$ to $M$ tending to the identity. Apart from the answer above, we propose the following formulation. We shall see that it implies relative semi-discreteness but we don't know whether the converse is true.

Definition 2.10. Let $N \subset M$ be a pair of von Neumann algebras with a given faithful normal conditional expectation $E: M \rightarrow N$. We say that $M$ is strongly semi-discrete relative to $N$ if there exists a net $\left(\phi_{i}\right)_{i \in I}$ of normal completely positive maps from $M$ to $M$ such that

i) $E \circ \phi_{i} \leq E \quad$ for all $i \in I$;

ii) $\phi_{i}$ is $N$-linear and $\phi_{i}(M)$ is contained in a finitely generated right $N$-submodule of $M$;

iii) $\quad\left(\phi_{i}(x)\right)$ converges $\sigma$-weakly to $x$ for all $x \in M$.

Proposition 2.11. Let $N \subset M$ be a pair of von Neumann algebras with a given faithful normal conditional expectation $E: M \rightarrow N$, such that $M$ is strongly semi-discrete relative to $N$. Then $M$ is semi-discrete relative to $N$.

Proof. Put $H={ }_{M} L^{2}(M)_{N}$. As explained in $\S 2.9$ above, $X=X(H)$ is the completion of $M$ endowed with the $N$-valued inner product $\left\langle m, m_{1}\right\rangle=$ $E\left(m^{*} m_{1}\right)$. Let $\phi: M \rightarrow M$ be a normal completely positive map satisfying properties i) and ii) of Definition 2.10. We shall prove that $\phi$ is a coefficient of the $M-M$ correspondence $X \otimes_{N} \bar{X}$. Then the condition iii) of Definition 2.10 will imply that the identity correspondence of $M$ is adherent to $\left\{X \otimes_{N} \bar{X}\right\}$.

Using the orthonormalization of Gram-Schmidt ([Rie2, Lemma 6.7]), we may find a finite orthonormal sequence $y_{1}, \ldots, y_{p}$ in $M$ such that $\phi(M) \subset$ 
$\sum_{i=1}^{p} y_{i} N$. Then for $x \in M$ we have

$$
\phi(x)=\sum_{i=1}^{p} y_{i} E\left(y_{i}^{*} \phi(x)\right) .
$$

Notice that $x \mapsto E\left(y_{i}^{*} \phi(x)\right)$ is a right $N$-linear form from $M$ into $N$ which is continuous since

$$
\begin{aligned}
{\left[E\left(y_{i}^{*} \phi(x)\right)\right]^{*} E\left(y_{i}^{*} \phi(x)\right) } & \leq E\left(\phi(x)^{*} y_{i} y_{i}^{*} \phi(x)\right) \\
& \leq\left\|y_{i}\right\|^{2} E\left(\phi(x)^{*} \phi(x)\right) \\
& \leq\|\phi(1)\|\left\|y_{i}\right\|^{2} E\left(\phi\left(x^{*} x\right)\right) \\
& \leq\|\phi(1)\|\left\|y_{i}\right\|^{2} E\left(x^{*} x\right)=\|\phi(1)\|\left\|y_{i}\right\|^{2}\langle x, x\rangle
\end{aligned}
$$

(see [Pas]). Therefore this form may be extended continuously to the selfdual completion $X$ of $M$ and there exists $\eta_{\imath} \in X$ such that

$$
E\left(y_{i}^{*} \phi(x)\right)=\left\langle\eta_{i}, x\right\rangle \quad \text { for all } x \in M \text {. }
$$

Thus $\phi(x)=\sum_{i=1}^{p} y_{i}\left\langle\eta_{i}, x\right\rangle=\langle\eta, x \xi\rangle$ with $\eta=\sum_{i} \eta_{i} \otimes y_{i}^{*}$ and $\xi=1 \otimes 1$. Since $\phi$ is self-adjoint, we have $\langle\eta, x \xi\rangle=\langle\xi, x \eta\rangle$ for all $x \in M$, and therefore

$$
\langle\xi+\eta, x(\xi+\eta)\rangle=\langle\xi, x \xi\rangle+\langle\eta, x \eta\rangle+2\langle\eta, x \xi\rangle .
$$

Put $\psi(x)=\langle\alpha, x \alpha\rangle$ with $\alpha=\frac{\xi+\eta}{\sqrt{2}}$. It follows that $\psi-\phi$ is a completely positive map, and by [Pas], there exists $T \in \mathcal{L}_{M}\left(X \otimes_{N} \bar{X}\right) \cap M^{\prime}$ such that $\phi(x)=\langle T \alpha, x T \alpha\rangle$ for all $x \in M$. This proves that $\phi$ is a coefficient of $X \otimes_{N} \bar{X}$.

Remarks 2.12. a) The hypothesis $E \circ \phi_{i} \leq E$ should be compared with Haagerup's result ([Haa3, Prop. 3.5]) showing in particular that in an injective $\mathrm{II}_{1}$ factor $M$ there is a net $\left(\phi_{i}\right)$ of normal finite rank completely positive maps with $\phi_{i}(1)=1$ and $\tau \circ \phi_{i}=\tau$ for all $i$ (where $\tau$ is the trace), which converges to the identity. The assumption $\tau \circ \phi_{i}=\tau$ is not necessary for $M$ to be injective. We do not know whether the inequality $E \circ \phi_{\imath} \leq E$ can be replaced by the condition $\phi_{i}(1) \leq 1$ in Definition 2.10 .

b) Let $N \subset M$ be a pair of von Neumann algebras, and let $A$ be the set of all $x \in M$ such that $N x N \cup N x^{*} N$ is contained in a finitely generated right $N$-submodule of $M$. Then $A$ is an involutive subalgebra of $M$ containing $N$. When $M$ is strongly semi-discrete relative to $N$, it is easily seen that $A$ is 
$\sigma$-weakly dense in $M$. In order to see whether semi-discrete implies strongly semi-discrete it would be interesting to see whether $A$ is $\sigma$-weakly dense in $M$, when $M$ is only supposed to be semi-discrete relative to $N$.

We end this section by two results which should be compared to analogous results of Bekka for group representations ([Bek, Corol. 5.4 and 5.6]).

Proposition 2.13. Let $M, N, P$ be von Neumann algebras, $H$ a left amenable correspondence from $M$ to $N$ and $K$ a left amenable correspondence from $N$ to $P$. Then $H \otimes_{N} K$ is a left amenable correspondence from $M$ to $P$.

Proof. Since ${ }_{N} L^{2}(N)_{N} \prec K \otimes_{P} \bar{K}$, it follows from Lemma 1.7 that

$$
{ }_{M} H \otimes_{N} L^{2}(N)_{N} \prec{ }_{M} H \otimes_{N} K \otimes_{P} \bar{K}_{N}
$$

and

$$
\begin{aligned}
{ }_{M} H \otimes_{N} \bar{H}_{M} & ={ }_{M} H \otimes_{N} L^{2}(N) \otimes_{N} \bar{H}_{M} \\
& \prec{ }_{M} H \otimes_{N} K \otimes_{P} \bar{K} \otimes_{N} \bar{H}_{M}={ }_{M}\left(H \otimes_{N} K\right) \otimes_{P}\left(\overline{H \otimes_{N} K}\right)_{M} .
\end{aligned}
$$

Therefore we have

$$
\operatorname{id}_{M} \prec{ }_{M} H \otimes_{N} \bar{H}_{M} \prec{ }_{M}\left(H \otimes_{N} K\right) \otimes_{P}\left(\overline{H \otimes_{N} K}\right)_{M} .
$$

Corollary 2.14. Let $N \subset M$ be a pair of von Neumann algebras such that ${ }_{M} L^{2}(M)_{N}$ is left and right amenable. If $H$ is a left amenable correspondence from $N$ to $N$, then $\operatorname{Ind}_{N}^{M} H$ is a left amenable correspondence from $M$ to $M$.

Proof. It is an immediate consequence of Proposition 2.13 since

$$
\operatorname{Ind}_{N}^{M} H={ }_{M} L^{2}(M) \otimes_{N} H \otimes_{N} L^{2}(M)_{M} .
$$

\section{Amenable and injective correspondences}

Definition 3.1. We say that a correspondence $H$ from $M$ to $N$ is left injective if there exists a norm one projection from $\mathcal{L}_{N^{0}}(H)$ onto $M$. In the case where $N$ is a von Neumann subalgebra of $M$ and $H={ }_{M} L^{2}(M)_{\dot{N}}$ we will rather say that $M$ is injective relative to $N$.

We begin by giving some examples of injective correspondences which come from group theory. Let us recall first the notion of amenability for an arbitrary unitary group representation introduced by Bekka in [Bek]. 
Definition 3.2. A unitary representation $\pi$ of a locally compact group $G$ on a Hilbert space $H$ is said to be amenable if there exists a state $\varphi$ on $\mathcal{L}(H)$ such that

$$
\varphi\left(\pi(s) T \pi\left(s^{-1}\right)\right)=\varphi(T) \quad \text { for all } s \in G \quad \text { and } T \in \mathcal{L}(H) .
$$

Such a state is called a $G$-invariant mean on $\mathcal{L}(H)$.

It was shown by Bekka ([Bek, Th. 5.1]) that $\pi$ is amenable if and only if the trivial representation $1_{G}$ of $G$ is weakly contained in the tensor product $\pi \otimes \bar{\pi}$, where $\bar{\pi}$ is the conjugate representation of $G$.

Amenable representations are closely related to injective correspondences as we will see now. Given a locally compact group $G$, its left reguilar representation will be denoted by $\lambda$, and $W^{*}(G)$ will be the von Neumann algebra generated by $\lambda(G)$. Recall that to a unitary representation $(\pi, H)$ of $G$ is associated a correspondence from $W^{*}(G)$ to $W^{*}(G)$ in the following way ([C-J]): the Hilbert space of the correspondence is $H \otimes L^{2}(G)$ and the left and right actions of $W^{*}(G)$ are well defined by

$$
\lambda(s) \xi \lambda(t)=(\pi(s) \otimes \lambda(s)) \xi(1 \otimes \lambda(t)) \quad \text { for all } s, t \in G, \xi \in H \otimes L^{2}(G) .
$$

Proposition 3.3. Let $(\pi, H)$ be a representation of a locally compact group $G$.

1) If $\pi$ is an amenable representation, the associated correspondence is left injective.

2) Suppose that $G$ is discrete. Then $\pi$ is an amenable representation if and only if the associated correspondence is left injective.

Proof. 1) Denote by $\alpha$ the action $s \mapsto \operatorname{Ad} \pi(s)$ from $G$ into $\mathcal{L}(H)$. By hypothesis, there is a state $\varphi$ on $\mathcal{L}(H)$ such that $\varphi \circ \alpha_{s}=\varphi$ for all $s \in G$. Passing to the crossed products, we get a norm one projection from $\mathcal{L}(H) \rtimes G$ onto $W^{*}(G)$ (identified with $1_{H} \otimes W^{*}(G)$ ) (see [AD1, Prop. 2.2]). Let $U$ be the unitary of $H \otimes L^{2}(G)$ defined by $(U \xi)(s)=\pi(s) \xi(s)$ for $\xi \in H \otimes L^{2}(G)$ and $s \in G$. Then $U(\mathcal{L}(H) \underset{\alpha}{\rtimes} G) U^{*}=\mathcal{L}(H) \otimes W^{*}(G)$, and $U\left(1_{H} \otimes W^{*}(G)\right) U^{*}$ is the von Neumann algebra generated by $\{\pi(s) \otimes \lambda(s), s \in G\}$. The existence of a norm one projection from $\mathcal{L}(H) \otimes W^{*}(G)$ onto $\{\pi(s) \otimes \lambda(s), s \in G\}^{\prime \prime}$ means exactly that $W_{W^{*}(G)} H \otimes L^{2}(G)_{W^{*}(G)}$ is a left injective correspondence.

2) Suppose now that $G$ is discrete, and that there exists a norm one projection from $\mathcal{L}(H) \otimes W^{*}(G)$ onto $\{\pi(s) \otimes \lambda(s), s \in G\}^{\prime \prime}$. Denote by $\tau$ the canonical trace on $\{\pi(s) \otimes \lambda(s), s \in G\}^{\prime \prime}$ (which is isomorphic to $W^{*}(G)$ ), 
and for $x \in \mathcal{L}(H)$, set $\varphi(x)=\tau \circ E\left(x \otimes 1_{L^{2}(G)}\right)$. Then, for $s \in G$ we have

$$
\begin{aligned}
\varphi(x \pi(s)) & =\tau \circ E\left(x \pi(s) \otimes 1_{L^{2}(G)}\right)=\tau \circ E\left[\left(x \otimes \lambda\left(s^{-1}\right)\right)(\pi(s) \otimes \lambda(s))\right] \\
& =\tau\left[E\left(x \otimes \lambda\left(s^{-1}\right)\right) \pi(s) \otimes \lambda(s)\right] \\
& =\tau \circ E\left(\pi(s) x \otimes 1_{L^{2}(G)}\right)=\varphi(\pi(s) x) .
\end{aligned}
$$

Let us consider now a dynamical system $(N, G, \alpha)$ and let us denote by $\bar{\alpha}$ the action of $G$ on $L^{\infty}(G) \otimes N$ obtained by tensoring the action by left translation on $L^{\infty}(G)$ and $\alpha$. We say that $(N, G, \alpha)$ is amenable if there exists a norm one projection $P: L^{\infty}(G) \otimes N \rightarrow N$ such that $P \circ \bar{\alpha}_{g}=\alpha_{g} \circ P$ for all $g \in G$ [AD1]. It is in particular the case when $G$ is an amenable group.

Proposition 3.4. Let $(N, G, \alpha)$ be a dynamical system.

1) If $(N, G, \alpha)$ is an amenable dynamical system, then $\underset{\alpha}{\rtimes} G$ is injective relative to $N$.

2) Suppose that $G$ is discrete. Then the following conditions are equivalent:

a) $N \rtimes G$ is injective relative to $N$;

b) $N^{\alpha} \underset{\alpha}{\alpha} G$ is amenable relative to $N$;

c) ${ }^{\alpha} \rtimes G$ is strongly semi-discrete relative to $N$;

d) $\stackrel{\alpha}{(N, G, \alpha)}$ is an amenable dynamical system.

Proof. 1) If $H$ is a standard form for $N$, then $K=L^{2}(G) \otimes H$ is a standard form for $M=N_{\alpha} G$, and $\mathcal{L}\left(L^{2}(G)\right) \otimes N=\mathcal{L}_{N^{0}}(K)$. Now 1) is obvious because the amenability of the dynamical system $(N, G, \alpha)$ implies the existence of a norm one projection from $\mathcal{L}\left(L^{2}(G)\right) \otimes N=\mathcal{L}_{N^{0}}(K)$ onto $M$ ([AD1, Prop. 3.11]).

2) Suppose now that $G$ is discrete. Each element $x \in M=N \rtimes G$ has a unique expression of the form $x=\sum_{s \in G} u_{s} x_{s}$, with $x_{s} \in N, u_{s}$ unitary, and $u_{s} n u_{s}^{*}=\alpha_{s}(n)$ for all $s \in G, n \in N$.

a) and d) are equivalent by ([AD1, Prop. 4.1]). Thanks to Propositions 2.11 and 2.5 we know also that $c) \Rightarrow b$ ) and b) $\Rightarrow$ a). It remains to show that $\mathrm{d}) \Rightarrow \mathrm{c})$. By $([\mathbf{A D 2}$, Th. 3.3]), if $(N, G, \alpha)$ is amenable, there exists a net $\left(h_{i}\right)$ of functions from $G$ into the centre $Z(N)$ of $N$, with finite support, of positive type with respect to $\alpha$ (see [AD2, Def. 2.1]), such that

$$
\left\{\begin{array}{l}
h_{i}(e) \leq 1 \quad \forall i \in I \\
\lim _{i} h_{i}(s)=1 \quad \sigma \text {-weakly, } \forall s \in G .
\end{array}\right.
$$


Denote by $\phi_{i}$ the normal completely positive map from $N \rtimes G$ to $N \rtimes G$ such $\phi_{i}\left(u_{s} x\right)=h_{i}(s) u_{s} x$ for all $s \in G$ and $x \in N$ (see [AD3, Prop. 2.8]). It is easily checked that $\left(\phi_{i}\right)_{i \in I}$ fulfils the conditions of Definition 2.10.

Let us come now to our last example. By a pair $G_{1} \subset G$ of locally compact groups, we mean a locally compact group $G$ and a closed subgroup $G_{1}$ of $G$. We choose a measure $\gamma$ on $G / G_{1}$, quasi-invariant by left translations, and denote by $\lambda_{G_{1}}$ the quasi-regular representation of $G$ in $L^{2}\left(G / G_{1}, \gamma\right)$. Recall that $W^{*}\left(G_{1}\right)$ appears in a natural way as a subalgebra of $W^{*}(G)$, and more precisely that we have $W^{*}\left(G_{1}\right)=W^{*}(G) \cap L^{\infty}\left(G / G_{1}\right)^{\prime}$, where $W^{*}(G)$ and $L^{\infty}\left(G / G_{1}\right)$ act in the obvious way on $L^{2}(G)([\mathbf{N}-\mathbf{T}])$. It is also useful to note that $L^{2}(G)$ is a standard form for $W^{*}(G)$.

The homogeneous space $G / G_{1}$ is said to be amenable if (among many other characterizations given by Eymard [Eym]) the following equivalent conditions hold :

1) there exists an invariant mean on $L^{\infty}\left(G / G_{1}\right)$;

2) the trivial representation of $G$ is weakly contained in $\lambda_{G_{1}}$. When $G_{1}$ is a normal subgroup of $G$ this means the quotient $G / G_{1}$ is an amenable group.

Proposition 3.5. Let $G_{1} \subset G$ be a pair of locally compact groups as above.

i) If the homogeneous space $G / G_{1}$ is amenable, then $W^{*}(G)$ is injective relative to $W^{*}\left(G_{1}\right)$.

ii) When $G$ is discrete, the converse is true.

Proof. i) The existence of an invariant mean on $L^{\infty}\left(G / G_{1}\right)$ implies the existence of a norm one projection from the crossed product $L^{\infty}\left(G / G_{1}\right) \rtimes G$ onto $W^{*}(G)$ (see [AD1, Prop. 2.2]). It is well known that $L^{\infty}\left(G / G_{1}\right) \rtimes G$ is Morita equivalent to $W^{*}\left(G_{1}\right)$. More precisely, there is a canonical isomorphism from $L^{\infty}\left(G / G_{1}\right) \rtimes G$ onto $\mathcal{L}_{W^{*}\left(G_{1}\right)^{0}}\left(L^{2}(G)\right)$ which preserves $W^{*}(G)$ (see for instance [AD5, Lemma 4.4]). This ends the proof of i).

ii) Let $E$ be a norm one projection from $\mathcal{L}_{W^{*}\left(G_{1}\right)^{0}}\left(L^{2}(G)\right)$ onto $W^{*}(G)$, and let $\tau$ be the canonical trace on $W^{*}(G)$. The restriction of $\tau \circ E$ to $L^{\infty}\left(G / G_{1}\right)$ gives an invariant mean on $L^{\infty}\left(G / G_{1}\right)$.

Let now $H$ be a correspondence from a von Neumann algebra $M$ to a von Neumann algebra $N$. We know that there exists a normal norm one projection from $\mathcal{L}_{N^{0}}(H)$ onto $M$ if and only if id $\operatorname{id}_{M}$ is contained in $H \otimes_{N} \bar{H}$ (see [AD5, Prop. 2.1]). By Proposition 2.5, we know also that if $\mathrm{id}_{M}$ is weakly contained in $H \otimes_{N} \bar{H}$, then there exists a norm one projection from $\mathcal{L}_{N^{0}}(H)$ onto $M$. We examine next the converse of this result. 
Proposition 3.6. Let $H$ be a left injective correspondence from $M$ to $N$. We suppose that there exists a faithful normal semi-finite weight $\varphi$ on $M$ such that, for all $t \in \mathbb{R}$, the automorphism $\sigma_{t}^{\varphi}$ is induced by a unitary of $\mathcal{L}_{N^{0}}(H)$, where $\sigma^{\varphi}$ denotes the modular automorphism group of $\varphi$. Then $H$ is a left amenable correspondence from $M$ to $N$.

The proof of this proposition follows the lines of the one given by A. Connes and $\mathrm{S}$. Wassermann in the case $N=\mathbb{C}$, which shows that an injective von Neumann algebra is semi-discrete $([\mathbf{C o 3}]$, [Was] $)$. Before going into the proof, let us indicate some examples where the assumption of the proposition is fulfilled.

First, it is the case for any correspondence ${ }_{M} H_{N}$ where $M$ is semi-finite. Secondly consider next the correspondence ${ }_{M} L^{2}(M)_{N}$ associated with a pair $N \subset M$ and suppose that there is a normal faithful semi-finite weight $\varphi$ on $M$ such that $\sigma_{t}^{\varphi}(x)=x$ for all $t \in \mathbb{R}$ and $x \in N$. For each $t$, denote by $v_{t}$ the canonical unitary of $L^{2}(M)$ which implements $\sigma_{t}^{\varphi}$. Since $v_{t}$ commutes with $N$ and $J_{M}$, we have $v_{t} \in \mathcal{L}_{N^{0}}\left(L^{2}(M)\right)$. Finally, let $M$ be a von Neumann algebra, $\varphi$ a normal faithful semi-finite weight on $M$, and set $N=M \underset{\sigma^{\varphi}}{\rtimes} \mathbb{R}$. Then the correspondence ${ }_{M} L^{2}(N)_{N}$ satisfies the required property.

The first step in the proof of Proposition 3.6 uses the following key lemma, due to A. Connes [Co3].

Lemma 3.7. Let $M \subset P$ be a pair of von Neumann algebras such that there exists a norm one projection from $P$ onto $M$. We suppose that a normal faithful finite trace $\tau$ is given on $M$. Then, for $\varepsilon>0$ and $b_{1}, \ldots, b_{n} \in M$, there exists a normal state $\varphi$ on $P$ with

$$
\left\|\left.\varphi\right|_{M}-\tau\right\| \leq \varepsilon \quad \text { and } \quad\left\|\left[b_{i}, \varphi\right]\right\| \leq \varepsilon, \quad 1 \leq i \leq n .
$$

Proof of Proposition 3.6. We suppose first that $M$ is finite, and we prove that if ${ }_{M} H_{N}$ is left injective, then the identity correspondence of $M$ is adherent to $\left\{H \otimes_{N} \bar{H}\right.$ \}. We have to show that, given $\varepsilon>0$ and $x_{1}, \ldots, x_{p}, y_{1}, \ldots, y_{q}$ in $M$, there exists $\xi \in H \otimes_{N} \bar{H}$ with

$$
\left|\left\langle\xi, x_{i} \xi y_{j}\right\rangle-\left\langle\xi_{0}, x_{i} \xi_{0} y_{j}\right\rangle\right| \leq \varepsilon \quad \text { for } i=1, \ldots, p, j=1, \ldots, q,
$$

where $\xi_{0}$ denotes the canonical vector in $L^{2}(M, \tau)$. There is no loss of generality to assume that $y_{1}, \ldots, y_{p}$ are unitaries. Let $\eta>0$ be given. By Lemma 3.7, we may choose a normal state $\varphi$ on $\mathcal{L}_{N^{0}}(H)$ with $\left\|\left.\varphi\right|_{M}-\tau\right\| \leq \eta$ and $\left\|\left[y_{i}, \varphi\right]\right\| \leq \eta$ for $i=1, \ldots, q$. Using the fact that $H \otimes_{N} \bar{H}$ is a standard form for $\mathcal{L}_{N^{0}}(H)$ ([S]), we may find a unique vector $\xi$ in the positive cone $\left(H \otimes_{N} \bar{H}\right)_{+}$such that $\varphi(x)=\langle\xi, x \xi\rangle$ for all $x \in \mathcal{L}_{N^{0}}(H)$ (see [Haa3]). Since 
$y_{i} \xi y_{i}^{*}$ is the vector of $\left(H \otimes_{N} \bar{H}\right)_{+}$associated with the state $y_{\imath} \varphi y_{i}^{*}$, by the Powers-Størmer inequality we get

$$
\left\|y_{i} \xi y_{i}^{*}-\xi\right\|^{2} \leq\left\|y_{i} \varphi y_{i}^{*}-\varphi\right\| \leq \eta
$$

and thus $\left\|y_{i} \xi-\xi y_{i}\right\| \leq \sqrt{\eta}$ for $i=1, \ldots, q$.

On the other hand, we know that $\left|\langle\xi, x \xi\rangle-\left\langle\xi_{0}, x \xi_{0}\right\rangle\right| \leq \eta\|x\|$ for all $x \in M$, from which it follows that

$$
\begin{aligned}
\mid\left\langle\xi, x_{i} \xi y_{j}\right\rangle-\left\langle\xi_{0}, x_{\imath} \xi_{0} y_{\jmath}\right\rangle & |\leq|\left\langle\xi, x_{\imath} \xi y_{j}\right\rangle-\left\langle\xi, x_{i} y_{j} \xi\right\rangle \mid \\
& +\left|\left\langle\xi, x_{\imath} y_{j} \xi\right\rangle-\left\langle\xi_{0}, x_{i} y_{j} \xi_{0}\right\rangle\right| \\
\leq & \left\|x_{i}\right\|\left\|y_{j} \xi-\xi y_{j}\right\|+\eta\left\|x_{i} y_{j}\right\| \leq\left\|x_{\imath}\right\| \sqrt{\eta}+\left\|x_{i} y_{j}\right\| \eta
\end{aligned}
$$

The conclusion follows immediately.

The next step consists in extending this result from the case $M$ finite to the case $M$ semi-finite. We may write $M=\prod_{i}\left(P_{i} \otimes \mathcal{L}\left(K_{i}\right)\right)$ where $P_{i}$ is a finite von Neumann algebra, and $\mathcal{L}\left(K_{i}\right)$ a type I factor. The techniques of reduction to the finite case are quite straightforward but lengthy and we shall omit the proof (see [Was] in the case $N=\mathbb{C}$ ).

The last step is a reduction to the semi-finite case, thanks to the use of crossed products. We denote by $\sigma$ instead of $\sigma^{\varphi}$ the modular automorphism group of $M$ and we set $P=M \rtimes \mathbb{R}, K=L^{2}(\mathbb{R}) \otimes H$. Let $\pi_{M}$ and $\pi_{N^{\circ}}$ be the left and right actions of $M$ and $N$ respectively on $H$. The canonical left action $\pi_{P}$ of $P$ on $K$ and the right action $1 \otimes \pi_{N^{0}}$ of $N$ endow $K$ with a structure of correspondence from $P$ to $N$. Note that for $x \in M$, we have

$$
\left(\pi_{P}(x) \xi\right)(t)=\pi_{M}\left(\sigma_{-t}(x)\right) \xi(\dot{t}) \quad \forall \xi \in K, \forall t \in \mathbb{R} .
$$

Moreover, we have $\mathcal{L}_{N^{0}}(K)=\mathcal{L}\left(L^{2}(\mathbb{R})\right) \otimes \pi_{N^{0}}\left(N^{0}\right)^{\prime}=\mathcal{L}\left(L^{2}(\mathbb{R})\right) \otimes \mathcal{L}_{N^{0}}(H)$.

Since ${ }_{M} H_{N}$ is left injective, there a norm one projection from $\mathcal{L}\left(L^{2}(\mathbb{R})\right) \otimes$ $\mathcal{L}_{N^{0}}(H)$ onto $\mathcal{L}\left(L^{2}(\mathbb{R})\right) \otimes M$, and since $\mathbb{R}$ is amenable there exists a norm one projection from $\mathcal{L}\left(L^{2}(\mathbb{R})\right) \otimes M$ onto $M \underset{\sigma}{\rtimes} \mathbb{R}$. It follows that ${ }_{P} K_{N}$ is a left injective correspondence and hence left amenable, because $P$ is semi-finite. By restriction we get, from the property $\operatorname{id}_{P} \prec{ }_{P} K \otimes_{N} \bar{K}_{P}$, that ${ }_{M} L^{2}(P)_{M} \prec$ ${ }_{M} K \otimes_{N} \bar{K}_{M}$. Then, by Proposition 2.6, we have $\operatorname{id}_{M} \prec{ }_{M} L^{2}(P)_{M}$, and therefore $\operatorname{id}_{M} \prec{ }_{M} K \otimes_{N} \bar{K}_{M}$.

Let us notice now that there is a canonical identification of

$$
K \otimes_{N} \bar{K}=\left(L^{2}(\mathbb{R}) \otimes H\right) \otimes_{N}\left(\overline{L^{2}(\mathbb{R})} \otimes \bar{H}\right)
$$

with $L^{2}(\mathbb{R}) \otimes L^{2}(\mathbb{R}) \otimes\left(H \otimes_{N} \bar{H}\right)$, and thus we view the elements of $K \otimes_{N} \bar{K}$ as functions with values in the Hilbert space $H \otimes_{N} \bar{H}$. The left and right 
actions of $P$ (resp. $M$ ) on $K \otimes_{N} \bar{K}$ (resp. $H \otimes_{N} \bar{H}$ ) will be denoted by $\rho_{P}$ and $\rho_{P^{0}}$ (resp. $\rho_{M}$ and $\rho_{M^{0}}$ ). For $x \in M$ and $\xi \in K \otimes_{N} \bar{K}$ we have

$$
\begin{gathered}
{\left[\rho_{P}(x) \xi\right](t, s)=\rho_{M}\left(\sigma_{-t}(x)\right)[\xi(t, s)]} \\
{\left[\rho_{P^{0}}(x) \xi\right](t, s)=\rho_{M^{0}}\left(\sigma_{-s}(x)\right)[\xi(t, s)] .}
\end{gathered}
$$

Let $x_{1}, \ldots, x_{k}, y_{1}, \ldots, y_{k}$ be given elements in $M$. Since $\operatorname{id}_{M} \prec{ }_{M} K \otimes_{N} \bar{K}_{M}$ we have

$$
\left\|\sum_{i} x_{i} J_{M} y_{i}^{*} J_{M}\right\| \leq\left\|\sum_{i} \rho_{P}\left(x_{i}\right) \rho_{P^{0}}\left(y_{i}\right)\right\|
$$

where $\sum_{i} \rho_{P}\left(x_{i}\right) \rho_{P^{0}}\left(y_{i}\right)$ is the decomposable operator

$$
(t, s) \mapsto \sum_{i} \rho_{M}\left(\sigma_{-t}\left(x_{i}\right)\right) \rho_{M^{0}}\left(\sigma_{-s}\left(y_{i}\right)\right) .
$$

Let us fix $(s, t) \in \mathbb{R} \times \mathbb{R}$. Since $\sigma_{-t}$ and $\sigma_{-s}$ are induced by unitaries in $\mathcal{L}_{N^{0}}(H)$, by [Co4] (see also [S, Lemme 2.3]), there is a unitary $U$ of $H \otimes_{N} \bar{H}$ such that

$$
\sum_{i} \rho_{M}\left(\sigma_{-t}\left(x_{i}\right)\right) \rho_{M^{0}}\left(\sigma_{-s}\left(y_{i}\right)\right)=U\left(\sum_{i} \rho_{M}\left(x_{i}\right) \rho_{M^{0}}\left(y_{i}\right)\right) U^{*}
$$

and therefore we get

$$
\left\|\sum_{i} \rho_{P}\left(x_{i}\right) \rho_{P^{0}}\left(y_{i}\right)\right\|=\left\|\sum_{i} \rho_{M}\left(x_{i}\right) \rho_{M^{0}}\left(y_{i}\right)\right\|
$$

and

$$
\left\|\sum_{i} \pi_{M}\left(x_{i}\right) \pi_{M^{0}}\left(y_{i}\right)\right\| \leq\left\|\sum_{i} \rho_{M}\left(x_{i}\right) \rho_{M^{0}}\left(y_{i}\right)\right\| .
$$

It follows that the identity correspondence of $M$ is weakly contained in $H \otimes_{N}$ $\bar{H}$.

Remark 3.8. In fact Proposition 3.6 remains true if we only suppose that the $\sigma_{t}$ 's belong to the closure $G$, in the group Aut $M$ gifted with its usual topology, of the group of all automorphisms of $M$ induced by unitaries $v \in \mathcal{L}_{N^{0}}(H)$ such that $\operatorname{Ad} v(M)=M$. This follows from the observation that the set of all automorphisms $\theta \in$ Aut $M$ such that

$$
\begin{aligned}
\left\|\sum_{i} \rho_{M}\left(\theta\left(x_{i}\right)\right) \rho_{M^{0}}\left(y_{i}\right)\right\| & =\left\|\sum_{i} \rho_{M}\left(x_{i}\right) \rho_{M^{0}}\left(y_{i}\right)\right\| \\
& =\left\|\sum_{i} \rho_{M}\left(x_{i}\right) \rho_{M^{0}}\left(\theta\left(y_{i}\right)\right)\right\|
\end{aligned}
$$


is closed in Aut $M$.

Unfortunately, (2) is not true for every $\theta \in$ Aut $M$ in general.

Corollary 3.9. Let $M \subset N$ be a pair of von Neumann algebras and assume that there exists a faithful semi-finite normal weight $\varphi$ on $M$ such that, for all $t \in \mathbb{R}$, the automorphism $\sigma_{t}^{\varphi}$ is induced by a unitary of $N$. Then the following conditions are equivalent:

1) There exists a norm one projection from $N$ onto $M$.

2) There exists a net $\left(\phi_{i}\right)$ of normal completely positive maps from $N$ to $M$, such that $\phi_{i}(1) \leq 1$ for all $i$, and $\lim _{i} \phi_{i}(x)=x \sigma$-weakly for all $x \in M$.

Proof. Apply Propositions 2.5 and 3.6 to the correspondence ${ }_{M} L^{2}(N)_{N}$.

Proposition 3.10. Let $M$ and $N$ be two von Neumann algebras. The following conditions are equivalent :

1) There exists a left injective correspondence from $M$ to $N$.

2) There exists a left amenable correspondence from $M$ to $N$.

Proof. 2) $\Rightarrow 1$ ) follows from Proposition 2.5. Conversely let $\left(H, \pi_{M}, \pi_{N^{0}}\right)$ be a left injective correspondence from $M$ to $N$, and choose a normal faithful semi-finite weight $\varphi$ on $M$. Consider the correspondence $\left(K, \gamma_{M}, \gamma_{N^{0}}\right)$ from $M$ to $N$, where $K=L^{2}(\mathbb{R}) \otimes H$ and

$$
\left(\gamma_{M}(x) \gamma_{N^{0}}(y) \xi\right)(t)=\pi_{M}\left(\sigma_{-t}(x)\right) \pi_{N^{0}}(y) \xi(t)
$$

for all $x \in M, \quad y \in N$ and $\xi \in L^{2}(\mathbb{R}) \otimes H$. We see from the proof of Proposition 3.6 that this correspondence is left amenable.

\section{Amenable equivalence of von Neumann algebras and applications}

Definition 4.1. Let $M$ and $N$ be two von Neumann algebras. We say that $M$ is amenably dominated by $N$, and we write $M \prec N$, if there exists a left amenable correspondence from $M$ to $N$. If $M \underset{a}{\prec} N$ and $N \underset{a}{\prec} M$, we will say that $M$ and $N$ are amenably equivalent, and write $\underset{a}{\sim} N$.

Using Proposition 3.10, we see that we may replace the word amenable by the word injective in the previous definition. Also Proposition 2.13 implies that $\prec$ is a preorder relation and that $\sim$ is actually an equivalence relation. 
Note that the relation $M \underset{a}{\prec} N$ means that there exists a von Neumann algebra $N_{1}$, Morita equivalent to $N$, containing $M$ as a von Neumann subalgebra and such that there exists a norm one projection from $N_{1}$ onto $M$. In particular, if $M$ is injective we have $M \prec N$ for all $N$.

\subsection{Examples of amenably equivalent von Neumann algebras.}

a) If $N$ is injective, we have $M \otimes N \sim M$ for all $M$ : obviously, since there is a norm one projection from $M \otimes N$ onto $M$, we have $M \underset{a}{\prec} M \otimes N$; on the other hand, $L^{2}(M) \otimes L^{2}(N)$ with its obvious left $M \otimes N$-action, and right $M$-action is left injective and therefore we have $M \otimes N \prec M$.

b) In [Po1, §1.4.3], S. Popa has defined two $\mathrm{II}_{1}$ factors $M$ and $N$ to be $w$-stable equivalent if there is a correspondence $H$ of finite index between them. This means that $\mathcal{L}_{N^{0}}(H)$ and $\mathcal{L}_{M}(H)$ are finite factors and thus there exists normal norm one projections from $\mathcal{L}_{N^{0}}(H)$ onto $M$ and from $\mathcal{L}_{M}(H)$ onto $\pi_{N^{0}}(N)$. Thus $H$ is amenable. Therefore, $w$-stable equivalence implies amenable equivalence, but the converse is not true because, for instance, property $T$ is preserved by $w$-stable equivalence but not by amenable equivalence.

c) Let $(N, G, \alpha)$ be a dynamical system. Then we have $N \underset{a}{\prec} \underset{\alpha}{\rtimes} G$ and if moreover the dynamical system is amenable, we have $N \underset{a}{\sim} \underset{\alpha}{{ }_{\alpha}^{a}} G$ (see Prop. 2.6 and Prop. 3.4).

d) Let $G_{1} \subset G$ be a pair of locally compact groups such that $G / G_{1}$ is an amenable homogeneous space. Then we have $W^{*}(G) \underset{a}{\prec} W^{*}\left(G_{1}\right)$, and if moreover $G$ is discrete, we have $W^{*}(G) \underset{a}{\sim} W^{*}\left(G_{1}\right)$. This follows from Proposition 3.5, and the fact that there exists a norm one projection from $W^{*}(G)$ onto $W^{*}\left(G_{1}\right)$ when $G$ is discrete.

e) Let $N$ be a von Neumann algebra and $\varphi$ a normal faithful semi-finite weight on $N$. We denote by $M$ the crossed product $N \rtimes \mathbb{R}$ of $N$ by the modular automorphism group $\sigma$ relative to $\varphi$. Example c) above shows that $N \underset{\sigma}{\rtimes} \underset{a}{\sim} N$, but here we may assert moreover that ${ }_{M} L^{2}(M)_{N}$ is a left and right amenable correspondence. In fact, we know by Proposition 3.4,1) that ${ }_{M} L^{2}(M)_{N}$ is left injective, hence left amenable, since $M$ is semi-finite (see Prop. 3.6). On the other hand, ${ }_{M} L^{2}(M)_{N}$ is right amenable by Proposition 2.6 .

Let us remark in particular that every von Neumann algebra is amenably equivalent to a semi-finite von Neumann algebra, and even to a semi-finite von Neumann algebra $M$ which is isomorphic to $M \otimes R$, where $R$ is the hyperfinite $\mathrm{II}_{1}$ factor.

We shall now prove that many weak form of amenability for von Neumann 
algebras are preserved by this equivalence relation. We need first to recall some definitions and results, mostly due to Uffe Haagerup ([Haa4], $[\mathbf{C}-\mathbf{H}]$ ).

Let $T$ be a bounded map from a $C^{*}$-algebra $A$ into a $C^{*}$-algebra $B$. For each integer $n \geq 1$, we denote by $T_{n}$ the map from $M_{n}(A)$ into $M_{n}(B)$ such that $T_{n}\left(\left[a_{i j}\right]\right)=\left[T\left(a_{i j}\right)\right]$. Then $T$ is called completely bounded if $\|T\|_{c b} \stackrel{\text { def }}{=}$ $\sup \left\|T_{n}\right\|<+\infty$.

$n \geq 1$

Definition 4.3 [Haa4]. a) We say that a von Neumann algebra $M$ has the weak ${ }^{*}$ completely bounded approximation property if there exist $c \in \mathbb{R}^{+}$ and a net of $\sigma$-weakly continuous finite rank operators $\left(\phi_{i}\right)_{i \in I}$ from $M$ to $M$ such that $\left\|\phi_{i}\right\|_{c b} \leq c$ for all $i$ and $\lim _{i} \phi_{\imath}(x)=x \sigma$-weakly for all $x \in M$. We let $\Lambda(M)$ be the infimum of all values of $c$ for which such nets exist. If $M$ has not this approximation property, we set $\Lambda(M)=+\infty$.

b) Similarly, a $C^{*}$-algebra $A$ is said to have the completely bounded approximation property if there exist $c \in \mathbb{R}^{+}$and a net of finite rank operators $\left(\phi_{i}\right)_{i \in I}$ from $A$ to $A$ such that $\left\|\phi_{i}\right\|_{c b} \leq c$ for all $i$ and $\lim _{i}\left\|\phi_{i}(x)-x\right\|=0$ for all $x \in A$. Then $\Lambda(A)$ is defined in the obvious way.

4.4. Let $G$ be a locally compact group and $A(G)$ its Fourier algebra, that is the predual of $W^{*}(G)$. The norm in $A(G)$ will be denoted by $\|\cdot\|_{A}$.

A multiplier $m$ of $A(G)$ is a bounded operator on $A(G)$ given by pointwise multiplication by a function on $G$. We say that $m$ is a completely bounded multiplier of $A(G)$ if the transposed operator on $W^{*}(G)$ is completely bounded, and then its complete bounded norm is denoted by $\|m\|_{M_{0}}$. Remark that every element $u$ in $A(G)$ is a completely bounded multiplier, and that we have $\|u\|_{M_{0}} \leq\|u\|_{A}$. For further details we refer the reader to $[\mathbf{d C H}]$ and $[\mathbf{C}-\mathbf{H}]$.

Following $[\mathbf{C - H}]$, we say that $G$ is weakly amenable if there exists a net $\left(u_{i}\right)_{i \in I}$ in $A(G)$ and $c \in \mathbb{R}^{+}$such that $\left\|u_{i}\right\|_{M_{0}} \leq c$ for all $i$ and $\lim _{i} u_{i}=1$ uniformly on compacts. We let $\Lambda_{G}$ be the infimum of all values of $c$ for which such nets exist, and we put $\Lambda_{G}=+\infty$ if $G$ is not weakly amenable. It is proved in $[\mathbf{C}-\mathbf{H}]$ that the existence of a net $\left(u_{i}\right)_{i \in I}$ as above is equivalent to the existence of an approximate unit $\left(v_{j}\right)_{j \in J}$ for $A(G)$ such that $\left\|v_{j}\right\|_{M_{0}} \leq c$ for all $j$. This definition may be compared to the well-known result of Leptin [Lep] asserting that $G$ is amenable if and only if it is possible to find an approximate unit $\left(v_{j}\right)$ for $A(G)$ such that $\left\|v_{j}\right\|_{A} \leq 1$ for all $j$. In particular, an amenable group $G$ is weakly amenable with $\Lambda_{G}=1$. The constant $\Lambda_{G}$ has been computed for all non-compact simple Lie groups with finite centre(see [dCH], [Cow], [Haa4], [C-H] for the computations and further details on the subject, and in particular the next proposition).

We denote, as usual, by $C_{r}^{*}(G)$ the reduced $C^{*}$-algebra of the group $G$, that is, the $C^{*}$-algebra generated by the left translation operators in $L^{2}(G)$. 
Proposition 4.5 ([Haa4, Th.2.6]). Let $G$ be a discrete group. Then the following conditions are equivalents :

a) $C_{r}^{*}(G)$ has the completely bounded approximation property.

b) $W^{*}(G)$ has the weak* completely bounded approximation property.

c) $G$ is weakly amenable.

Moreover $\Lambda\left(C_{r}^{*}(G)\right)=\Lambda\left(W^{*}(G)\right)=\Lambda_{G}$.

Lemma 4.6. Let $N$ be a reduced von Neumann algebra of $M$. Then we have $\Lambda(N) \leq \Lambda(M)$.

Proof. Let $e$ be the projection in $M$ such that $N=e M e$ and denote by $E$ the map $x \mapsto e x e$ from $M$ onto $N$. Then, if $\left(\phi_{i}\right)_{i \in I}$ is a net of $\sigma$-weakly continuous finite rank operators on $M$, as in Definition $4.3 \mathrm{a}),\left(E \circ \phi_{i}\right)_{i \in I}$ defines, by restriction, a net $\left(\psi_{i}\right)_{i \in I}$ of $\sigma$-weakly continuous finite rank operators on $N$, with $\sup _{i}\left\|\psi_{i}\right\|_{c b} \leq \sup _{i}\left\|\phi_{i}\right\|_{c b}$ and $\lim _{i} \psi_{i}(x)=x \sigma$-weakly for all $x \in$ $N$.

Lemma 4.7. Let $N$ and $M$ be two von Neumann algebras. Then we have $\Lambda(M \otimes N) \leq \Lambda(M) \Lambda(N)$.

Proof. We may suppose that $\Lambda(M)<+\infty$ and $\Lambda(N)<+\infty$. Given $\varepsilon>0$, by convexity arguments we see that there exist nets $\left(\phi_{i}\right)_{i \in I}$ and $\left(\psi_{j}\right)_{j \in J}$ of $\sigma$-weakly continuous finite rank operators on $M$ and $N$ respectively, with

$$
\sup _{i}\left\|\phi_{i}\right\|_{c b} \leq \Lambda(M)+\varepsilon, \quad \sup _{i}\left\|\psi_{i}\right\|_{c b} \leq \Lambda(N)+\varepsilon,
$$

and

$\lim _{i}\left\|\varphi \circ \phi_{i}-\varphi\right\|=0, \quad \lim _{j}\left\|\psi \circ \phi_{j}-\psi\right\|=0 \quad$ for all $\varphi \in M_{*}, \psi \in N_{*}$.

By ([C-H, Lemma 1.5]), for $(i, j) \in I \times J$, there is a unique $\sigma$-weakly continuous map, denoted by $\phi_{i} \otimes \psi_{j}$, from $M \otimes N$ onto itself, such that $\left(\phi_{i} \otimes \psi_{j}\right)(x \otimes y)=\phi_{i}(x) \otimes \psi_{j}(y)$ for $x \in M$ and $y \in N$, and we have

$$
\left\|\phi_{i} \otimes \psi_{j}\right\|_{c b} \leq\left\|\phi_{i}\right\|_{c b}\left\|\psi_{j}\right\|_{c b} .
$$

Obviously, $\phi_{i} \otimes \psi_{j}$ has a finite rank, and for $\varphi \in M_{*}, \psi \in N_{*}$, we get

$$
\lim _{i, j}\left\|\left(\varphi \circ \phi_{i}\right) \otimes\left(\psi \circ \psi_{j}\right)-\varphi \otimes \psi\right\|=0 .
$$

Since $M_{*} \odot N_{*}$ is norm dense in the predual of $M \otimes N$, it follows thàt $\left(\phi_{i} \otimes \psi_{j}\right)_{i, j}$ converges to the identity map of $M \otimes N$ in the topology of $\sigma$-weak pointwise convergence. Then we have

$$
\Lambda(M \otimes N) \leq(\Lambda(M)+\varepsilon)(\Lambda(N)+\varepsilon) \text { for all } \varepsilon>0,
$$


and thus $\Lambda(M \otimes N) \leq \Lambda(M) \Lambda(N)$.

It does not seem to be known whether $\Lambda(M \otimes N)=\Lambda(M) \Lambda(N)$ in general (see $[\mathbf{C - H}]$, last remark).

Lemma 4.8. If $N_{1}$ and $N_{2}$ are two Morita equivalent von Neuman algebras, we have $\Lambda\left(N_{1}\right)=\Lambda\left(N_{2}\right)$.

Proof. Since $N_{1}$ is isomorphic to a reduced von Neumann algebra of $N_{2} \otimes F$ where $F$ is a type I factor, it follows from Lemmas 4.6 and 4.7 that $\Lambda\left(N_{1}\right) \leq$ $\Lambda\left(N_{2}\right)$. In the same way we get $\Lambda\left(N_{2}\right) \leq \Lambda\left(N_{1}\right)$.

Theorem 4.9. Let $M$ and $N$ be two von Neumann algebras such that $M \prec \underset{a}{\prec}$. Then we have $\Lambda(M) \leq \Lambda(N)$.

Proof. Let $H$ be a left amenable correspondence from $M$ to $N$, and put $N_{1}=\mathcal{L}_{N^{0}}(H)$. Then $N_{1}$ is Morita equivalent to $N$ and we have (see $\S 2.1$ )

$$
\operatorname{id}_{M} \prec{ }_{M} H \otimes_{N} \bar{H}_{M}={ }_{M} L^{2}\left(N_{1}\right)_{M}={ }_{M} L^{2}\left(N_{1}\right) \otimes_{N_{1}} L^{2}\left(N_{1}\right)_{M} .
$$

Since $\Lambda\left(N_{1}\right)=\Lambda(N)$, and writing $N$ instead of $N_{1}$, we are reduced to proving that, when $M$ is a von Neumann subalgebra of $N$ with ${ }_{M} L^{2}(N)_{N}$ left amenable, then we have $\Lambda(M) \leq \Lambda(N)$. Using Proposition 2.5, we know that there exists a net $\left(\phi_{i}\right)_{i \in I}$ of normal completely positive maps from $N$ to $M$, such that $\phi_{i}(1) \leq 1$ for all $i \in I$, and $\lim _{i} \phi_{i}(x)=x \sigma$-weakly for all $x \in M$.

On the other hand, given $\varepsilon>0$, let $\left(\psi_{j}\right)_{j \in J}$ be a net of $\sigma$-weakly continuous finite rank operators on $N$, with $\sup _{j}\left\|\psi_{j}\right\|_{c b} \leq \Lambda(N)+\varepsilon$, and which tends to the identity map of $N$ in the topology of pointwise $\sigma$-weak convergence. Let us denote by $\theta_{i, j}$ the map $x \mapsto \phi_{i} \circ \psi_{j}(x)$ from $M$ into $M$. Obviously $\theta_{i, j}$ is $\sigma$-weakly continuous with finite rank, and we have

$$
\left\|\theta_{i, j}\right\|_{c b} \leq\left\|\phi_{i}\right\|_{c b}\left\|\psi_{j}\right\|_{c b} \leq\|\Lambda(N)\|+\varepsilon \text {. }
$$

Let $\eta>0, x_{1}, \ldots, x_{s} \in M, \varphi_{1}, \ldots, \varphi_{r} \in M_{*}$ be given. For $l=1, \ldots, r$ and $m=1, \ldots, s$ we have

$$
\left|\varphi_{l}\left(\theta_{i, j}\left(x_{m}\right)-x_{m}\right)\right| \leq\left|\varphi_{l}\left[\phi_{i}\left(\psi_{j}\left(x_{m}\right)-x_{m}\right)\right]\right|+\left|\varphi_{l}\left(\phi_{i}\left(x_{m}\right)-x_{m}\right)\right| .
$$

First, we may choose $i$ such that $\left|\varphi_{l}\left(\phi_{i}\left(x_{m}\right)-x_{m}\right)\right| \leq \eta / 2$ for all $l$ and $m$. Then, we may choose $j$ such that $\left|\varphi_{l}\left[\phi_{i}\left(\psi_{j}\left(x_{m}\right)-x_{m}\right)\right]\right| \leq \eta / 2$ for all $l$ and $m$. It follows that the identity map of $M$ is the pointwise $\sigma$-weak limit of a net $\left(\theta_{\lambda}\right)$ of $\sigma$-weakly continuous finite rank maps on $M$, with $\left\|\theta_{\lambda}\right\|_{c b} \leq \Lambda(N)+\varepsilon$. Thus we have $\Lambda(M) \leq \Lambda(N)$. 
4.10. Let us now list some immediate consequences of the previous theorem.

a) Suppose that $M$ is a von Neumann subalgebra of $N$ such that there is a norm one projection from $N$ onto $M$. Then we have $M \prec N$ and therefore $\Lambda(M) \leq \Lambda(N)$. Note that the only difficulty in this result is that the projection is not supposed to be normal.

b) Let $M \subset N$ be an inclusion of finite $\mathrm{II}_{1}$ factors with finite index [ $N$ : $M]$. Then $\Lambda(M)=\Lambda(N)$ (see $\S 4.2 \mathrm{~b}$ ).

c) Let $(M, G, \alpha)$ de a dynamical system. Then we have $\Lambda(M) \leq \Lambda(M \rtimes G)$, and $\Lambda(M)=\Lambda\left(\underset{\alpha}{\left.M_{\alpha} G\right)}\right.$ if moreover the dynamical system is supposed to be amenable (see $\S 4.2 \mathrm{c}$ ).

d) Let $G_{1} \subset G$ be a pair of locally compact groups such that $G / G_{1}$ is an amenable homogeneous space. Then we have $\Lambda\left(W^{*}(G)\right) \leq \Lambda\left(W^{*}\left(G_{1}\right)\right)$. If moreover $G$ is discrete, we have $\Lambda\left(W^{*}(G)\right)=\Lambda\left(W^{*}\left(G_{1}\right)\right.$ ) (see $\S 4.2$ d).

In particular, if $1 \rightarrow G_{1} \rightarrow G \rightarrow H \rightarrow 0$ is an exact sequence of discrete groups where $H$ is an amenable group, we see that $\Lambda_{G}=\Lambda_{G_{1}}$. This result should be compared to the following example. Let $G$ be the semi-direct product of $G_{1}=\mathbb{Z}^{2}$ by $H=S L(2, \mathbb{Z})$ under the natural action of $S L(2, \mathbb{Z})$ on $\mathbb{Z}^{2}$. Then U. Haagerup has shown [Haa4] that $\Lambda_{G}=+\infty$, although $G_{1}$ and $H$ are weakly amenable with $\Lambda_{G_{1}}=\Lambda_{H}=1$.

It would be interesting to see whether $\Lambda_{G}=\Lambda_{G_{1}}$ for any pair of locally compact groups $G_{1} \subset G$ such that $G / G_{1}$ is an amenable space. This would require of course a direct proof, not using the von Neumann algebra setting. Let us note that Haagerup proved the equality $\Lambda_{G}=\Lambda_{G_{1}}$ when $G_{1}$ is a lattice in $G$.

Almost exactly as in the proof of Theorem 4.9, we may show that the approximation properties described below (Def. 4.11, 4.12, 4.13) are transmitted from a von Neumann algebra $N$ to any von Neumann algebra $M$ such that $M \prec N$, and therefore are an invariant of amenable equivalence. We will only sketch the proof for the last one.

Recall first that a linear map $T$ from a $C^{*}$-algebra $A$ to a $C^{*}$-algebra $B$ is said to be $n$-positive, where $n$ is an integer $\geq 1$, if $T_{n}: M_{n}(A) \rightarrow M_{n}(B)$, defined by $T_{n}\left(\left[a_{i j}\right]\right)=\left[T\left(a_{i j}\right)\right]$ is positive.

Definition 4.11. We say that a von Neumann algebra $M$ has the weak* $n$-positive approximation property if there exists a net $\left(\phi_{i}\right)$ of $\sigma$-weakly continuous $n$-positive, finite rank operators from $M$ to $M$ such that $\phi_{i}(1)=1$ for all $i$ and $\lim _{i} \phi_{i}(x)=x \sigma$-weakly for all $x \in M$.

This property is stable by amenable equivalence. In $[\mathbf{d C H}]$ it is proved that the von Neumann algebras of the free groups have the weak ${ }^{*} n$-positive 
approximation property for all $n \geq 1$ and therefore it is also the case, for instance, for $W^{*}(S L(2, \mathbb{Z}))$.

The second approximation property which can be shown to be stable by amenable equivalence is the following one, recently introduced by J. Kraus $[\mathbf{K}]$ in connection with the slice map problem.

Definition 4.12. Let $M$ be a von Neumann algebra and $K$ an infinite dimensional Hilbert space. We say that $M$ has the weak* complete pointwise approximation property (CPWAP) if there is a net $\left(\phi_{\imath}\right)$ of $\sigma$-weakly continuous finite rank maps from $M$ to $M$ such that, for all $x \in M \otimes \mathcal{L}(K)$, we have $\lim _{i}\left(\phi_{i} \otimes 1_{\mathcal{L}(K)}\right)(x)=x \sigma$-weakly.

It is easily seen that if $M$ has the weak* completely bounded approximation property, then $M$ has the CPWAP, but the converse is not true (see [K, Example 2.11]). Kraus also proved that not every von Neumann algebra has the CPWAP. In $[\mathbf{H}-\mathbf{K}]$, it is shown that the class of discrete groups whose von Neumann algebra has the CPWAP is closed under taking semidirect products. Since CPWAP is invariant by amenable equivalence, we obtain here that, whenever $G_{1} \subset G$ is a pair of discrete groups such that $G / G_{1}$ is an amenable homogeneous space, then $W^{*}\left(G_{1}\right)$ has the CPWAP if and only if $W^{*}(G)$ has the same property.

We come now to our last example of approximation property which is invariant under amenable equivalence. For group von Neumann algebras, it is a property which appears for the first time in the work of Haagerup [Haa2], and is now known as Haagerup's approximation property.

Definition 4.13. We say that a von Neumann algebra $M$ satisfies the compact approximation property if there exists a net $\left(\phi_{i}\right)_{i \in I}$ of $\sigma$-weakly continuous completely positive maps from $M$ to $M$ such that

i) for all $x \in M$ we have $\lim _{i} \phi_{i}(x)=x \quad(\sigma$-weakly $)$;

ii) for all $\xi \in L^{2}(M)$ and $i \in I$, the map $x \mapsto \phi_{i}(x) \xi$ is compact from the normed space $M$ to $L^{2}(M)$.

Remark 4.14. Obviously, the above property ii) does not depend on the choice of a standard form $L^{2}(M)$ for $M$. Moreover it is enough to check it for $\xi$ in a total subset of $L^{2}(M)$. Let for instance $\xi_{0}$ be a vector in $L^{2}(M)$ such that $M^{\prime} \xi_{0}$ is dense in $L^{2}(M)$. Suppose that $x \mapsto \phi_{i}(x) \xi_{0}$ is compact. Then for all $y \in M^{\prime}$ the map $x \mapsto \phi_{i}(x) y \xi_{0}=y \phi_{i}(x) \xi_{0}$ is compact. Therefore in this case it suffices to check property ii) only for $\xi=\xi_{0}$

Definition 4.15. a) Let $M$ be a finite von Neumann algebra equipped with a normal faithful trace $\tau$ on $M$. We say that $M$ satisfies Haagerup's approximation property if there exists a net $\left(\phi_{i}\right)$ of $\sigma$-weakly continuous completely positive maps from $M$ to $M$ such that

i) $\tau \circ \phi_{i}\left(x^{*} x\right) \leq \tau\left(x^{*} x\right)$ for all $x \in M$; 
ii) $\lim _{i}\left\|\phi_{i}(x)-x\right\|_{2}=0$ for all $x \in M$;

iii) each $\phi_{i}$ induces a compact bounded operator on $L^{2}(M)$.

b) Let $G$ be a discrete group. We say that $G$ satisfies Haagerup's approximation property if there exists a net $\left(\varphi_{i}\right)_{i}$ of positive type functions on $G$ such that

i) $\lim _{i} \varphi_{i}(s)=1$ for all $s \in G$.

ii) each $\varphi_{i}$ vanishes at infinity.

Proposition 4.16. Let $G$ be a discrete group, and denote by $\tau$ the canonical trace on $W^{*}(G)$. Then the following conditions are equivalent:

a) $W^{*}(G)$ satisfies the compact approximation property;

b) $W^{*}(G)$ satisfies Haagerup's approximation property;

c) G satisfies Haagerup's approximation property.

Proof. The proof may be found almost entirely in [Cho]. Put $M=W^{*}(G)$. To show a) $\Rightarrow$ c) take a net $\left(\phi_{i}\right)_{i \in I}$ as in Definition 4.13. For $i \in I$, let $\varphi_{i}$ be the function $s \mapsto \tau\left(\phi_{i}(\lambda(s)) \lambda(s)^{*}\right)$ defined on $G$. Then $\left(\varphi_{i}\right)_{i \in I}$ is a net of positive type functions on $G$ such that $\lim _{i} \varphi_{i}(s)=1$ for all $s \in G$.

Consider $\phi: M \rightarrow M$ such that $T_{\phi}: x \mapsto \phi(x) \xi_{\tau}$ is a compact operator from $M$ to $L^{2}(M)$ and put $\varphi(s)=\tau\left(\phi(\lambda(s)) \lambda(s)^{*}\right)$ for $s \in G$ (where $\xi_{\tau}$ denotes the canonical vector in $L^{2}(M)=L^{2}(M, \tau)$ ). It remains to check that $\varphi$ vanishes at infinity. Given $\varepsilon>0$, let $T: M \mapsto L^{2}(M)$ be a bounded finite rank operator with $\left\|T_{\phi}-T\right\| \leq \varepsilon$. Since we have

$$
|\varphi(s)-\langle\lambda(s), T(\lambda(s))\rangle| \leq\left\|T_{\phi}(\lambda(s))-T(\lambda(s))\right\|_{2} \leq \varepsilon, \quad \text { for all } \quad s \in G,
$$

it is enough to prove that $s \mapsto\langle\lambda(s), T(\lambda(s))\rangle$ vanishes to infinity. But this is obvious because $\{\lambda(s), s \in G\}$ is an orthonormal basis of $L^{2}(M)$ and $T$ is a bounded finite rank map.

For the equivalence between b) and c) see [Cho].

b) $\Rightarrow$ a) Let $\left(\phi_{i}\right)$ be a net as in Definition 4.15. Since the injection $M \rightarrow$ $L^{2}(M)$ is bounded, we see that $x \mapsto \phi_{i}(x) \xi_{\tau}$ is a compact map from $M$ to $L^{2}(M)$. Then property ii) of Definition 4.13 follows from Remark 4.14. On the other hand property i) in Definition 4.13 is an immediate consequence of ii) in Definition 4.15.

Proposition 4.17. Let $M$ and $N$ be two von Neumann algebras. We suppose that $N$ has the compact approximation property and that $M$ is amenàbly dominated by $N$. Then $M$ has also the compact approximation property.

Proof. As for the proof of Theorem 4.9, we show first, by straightforward arguments, that the compact approximation property is invariant by Morita 
equivalence. Thus we may suppose that $M$ in contained in $N$ in such a way that there exists a net $\left(\phi_{i}\right)_{i \in I}$ of completely positive maps from $N$ to $M$ satisfying conditions ii) of Proposition 2.4, with $N_{1}$ replaced by $N$. Let $\left(\psi_{j}\right)_{j \in J}$ be a net of $\sigma$-weakly continuous maps from $N \rightarrow N$ converging to the identity map, and such that $x \mapsto \psi_{j}(x) \xi$ is a compact map from $N$ to $L^{2}(N)$ for all $j \in J$ and $\xi \in L^{2}(N)$. We have to check that $x \mapsto \phi_{i} \circ \psi_{\jmath}(x) \xi$ is a compact map from $M$ to $L^{2}(M)$. But this is obvious since each $\phi_{i}$ is a finite sum of maps of the form $x \mapsto W^{*} x W$ with $W \in \operatorname{Hom}_{M^{0}}\left(L^{2}(M), L^{2}(N)\right)$.

Remark 4.18. We may show, as in [Rob], that a type $\mathrm{II}_{1}$ factor with property $T$ cannot have the compact approximation property (see also $[\mathbf{C}-\mathbf{J}]$ ). Therefore such a factor cannot be amenably dominated by a von Neumann algebra with the compact approximation property.

\section{References}

[AD1] C. Anantharaman-Delaroche, Action moyennable d'un groupe localement compact sur une algebre de von Neumann, Math. Scand., 45 (1979), 289-304.

[AD2] C. Anantharaman-Delaroche, Systèmes dynamiques non commutatifs et moyennabilité, Math. Ann., 279 (1987), 297-315.

[AD3] C. Anantharaman-Delaroche, On relatıve amenability for von Neumann algebras, Compositio Math., 74 (1990), 333-352.

[AD4] C. Anantharaman-Delaroche, J.F. Havet, Approximate factorizations of completely positive maps, J. Funct. Anal., 90 (1990), 411-428.

[AD5] C. Anantharaman-Delaroche, Atomic correspondences, Preprint, Orléans (1991).

[BDH] M. Baillet, Y. Denizeau and J.F. Havet, Indice d'une espérance conditionnelle, Compositio Math., 66 (1988), 199-236.

[Bek] M. Bekka, Amenable unitary representations of locally compact groups, Invent. Math., 100 (1990), 383-401.

$[\mathrm{dCH}]$ J. de Canniere and U. Haagerup, Multipliers of the Fourier algebra of some simple Lie groups and their discrete subgroups, Amer. J. Math,. 107 (1984), 455-500.

[Cho] M. Choda, Group factors of the Haagerup type, Proc. Japan Acad., 59 (1983), 174-177.

[Co1] A. Connes, Une classification des facteurs de type III, Ann. Scient. Ec. Norm. Sup., 6 (1973), 133-252.

[Co2] A. Connes, Classification of injective factors, Ann. of Math., 104 (1976), 73-115.

[Co3] A. Connes, On the equivalence between injectivity and semi discreteness for operator algebras, Algèbres d'opérateurs et leurs applications en physique mathématique, Colloques Internationaux du C.N.R.S., No 274 (Marseille 1977), 107-112.

[Co4] A. Connes, Classification des facteurs, Proc. Symp. Pure Math., 38-2 (Amer. Math. Soc. 1982), 42-109.

[Co5] A. Connes, Correspondences, Unpublished.

[C-J] A. Connes and V. Jones, Property $T$ for von Neumann algebras, Bull. London Math., Soc., 17 (1985), 57-62. 
[Cow] M. Cowling, Harmonic analysis on some nilpotent groups (with applications to the representation theory of some semisimple Lie groups), Topics in Modern Harmonic Analysis and Group Representations, Vol. I, Istituto Nazionale di Alta Matematica (Roma 1983) 81-123.

[C-H] M. Cowling and U. Haagerup, Completely bounded multipliers of the Fourier algebra of a simple Lie group of real rank one, Invent. Math., 96 (1989), 507-549.

[E-L] E.G. Effros and E.C. Lance, Tensor products of operator algebras, Adv. in Math., 25 (1977), 1-34

[Eym] P. Eymard, Moyennes invariantes et représentation unitaires, Lecture Notes in Math., No 300, Springer-Verlag, New-York, Heidelberg, Berlin, 1972.

[Fel] J.M.G. Fell, Weak containement and induced representations of groups, Canad. J. Math., 14 (1962), 237-268.

[Haa1] U. Haagerup, The standard form of von Neumann algebras, Math. Scand., 37 (1975), 271-283.

[Haa2] U. Haagerup, An example of non-nuclear $C^{*}$-algebra which has the metric approximation property, Invent. Math., 50 (1979), 279-293.

[Haa3] U. Haagerup, A new proof of the equivalence of injectivity and hyperfiniteness for factors on a separable Hilbert space, J. Funct. Anal., 62 (1985), 160-201.

[Haa4] U. Haagerup, Group $C^{*}$-algebras without the completely bounded approximation property, Unpublished manuscript (1986).

[K] J. Kraus, The slice map problem and approximation property, J. Funct. Anal., 102 (1991), 116-155

[H-K] U. Haagerup and J. Kraus, Approximations properties for group $C^{*}$-algebras and group von Neumann algebras, Preprint.

[Lep] H. Leptin, Sur l'algèbre de Fourier d'un groupe localement compact, C. R. Acad. Sci. Paris, Série, A, 266 (1968), 1180-1182.

[N-T] Y. Nagakami and M. Takesaki, Duality for crossed products of von Neumann algebras, Lecture Notes in Math., No 731, Springer-Verlag, New-York, Heidelberg, Berlin, 1979.

[Pas] W.L. Paschke, Inner product modules over B*-algebras, Trans. Amer. Math. Soc., 182 (1973), 443-468.

[Po1] S. Popa, Correspondences, Preprint INCREST (1986).

[Po2] S. Popa, Classification of amenable subfactors of type II, Preprint, IHES (1992).

[Rie1] M.A. Rieffel, Induced representations of $C^{*}$-algebras, Advances in Math., 13 (1974), 176-257.

[Rie2] M.A. Rieffel, Morita equivalence for $C^{*}$-algebras and $W^{*}$-algebras, J. Pure Appl. Alg., 5 (1974), 51-96.

[Rob] A.G. Robertson, Strong non-amenability of $\mathrm{II}_{1}$ factors with property $T$, Bull. London Math. Soc., 20 (1988), 51-53.

[S] J.L. Sauvageot, Sur le produit tensoriel relatif d'espaces de Hilbert, J. Funct. Anal., 74 (1983), 305-319. 
[Was] S. Wasserman, Injective $W^{*}$-algebras, Math. Proc. Cambridge Phil. Soc., 82 (1977), 39-47.

Received March 30, 1993 and revised September 20, 1993.

UNIVERSITE D'ORLEANS

B.P. 6759, 45067 Orleans Cedex 2

FRANCE

E-mail address: claire@talcy.univ-orleans.fr 



\title{
PACIFIC JOURNAL OF MATHEMATICS
}

Founded by E. F. Beckenbach (1906-1982) and F. Wolf (1904-1989)

\section{EDITORS}

\author{
Sun-Yung Alice Chang (Managing Editor) \\ University of California \\ Los Angeles, CA 90095-1555 \\ pacific@math.ucla.edu
}

\section{F. Michael Christ}

University of California

Los Angeles, CA 90095-1555

christ@math.ucla.edu

Thomas Enright

University of California

San Diego, La Jolla, CA 92093

tenright@ucsd.edu

Nicholas Ercolani

University of Arizona

Tucson, AZ 85721

ercolani@math.arizona.edu
Robert Finn

Stanford University

Stanford, CA 94305

finn@gauss.stanford.edu

Vaughan F. R. Jones

University of California

Berkeley, CA 94720

vfr@math.berkeley.edu

Steven Kerckhoff

Stanford University

Stanford, CA 94305

spk@gauss.stanford.edu
Martin Scharlemann

University of California

Santa Barbara, CA 93106

mgscharl@math.ucsb.edu

Gang Tian

Courant Institute

New York University

New York, NY 10012-1100

tiang@taotao.cims.nyu.edu

V. S. Varadarajan

University of California

Los Angeles, CA 90095-1555

vsv@math.ucla.edu

\section{SUPPORTING INSTITUTIONS}

\section{CALIFORNIA INSTITUTE OF TECHNOLOGY \\ NEW MEXICO STATE UNIVERSITY \\ OREGON STATE UNIVERSITY \\ STANFORD UNIVERSITY \\ UNIVERSITY OF ARIZONA \\ UNIVERSITY OF BRITISH COLUMBIA \\ UNIVERSITY OF CALIFORNIA UNIVERSITY OF HAWAII}

\author{
UNIVERSITY OF MONTANA \\ UNIVERSITY OF NEVADA, RENO \\ UNIVERSITY OF OREGON \\ UNIVERSITY OF SOUTHERN CALIFORNIA \\ UNIVERSITY OF UTAH \\ UNIVERSITY OF WASHINGTON \\ WASHINGTON STATE UNIVERSITY
}

The supporting Institutions listed above contribute to the cost of publication of this Journal, but they are not owners or publishers and have no responsibility for its contents or policies.

Manuscripts must be prepared in accordance with the instructions provided on the inside back cover.

The Pacific Journal of Mathematics (ISSN 0030-8730) is published monthly except for July and August. Regular subscription rate: $\$ 215.00$ a year (10 issues). Special rate: $\$ 108.00$ a year to individual members of supporting institutions.

Subscriptions, orders for back issues published within the last three years, and changes of subscribers address should be sent to Pacific Journal of Mathematics, P.O. Box 4163, Berkeley, CA 94704-0163, U.S.A. Prior back issues are obtainable from Kraus Periodicals Co., Route 100, Millwood, NY 10546.

The Pacific Journal of Mathematics at the University of California, c/o Department of Mathematics, 981 Evans Hall, Berkeley, CA 94720 (ISSN 0030-8730) is published monthly except for July and August. Second-class postage paid at Berkeley, CA 94704, and additional mailing offices. POSTMASTER: send address changes to Pacific Journal of Mathematics, P.O. Box 6143, Berkeley, CA 94704-0163.

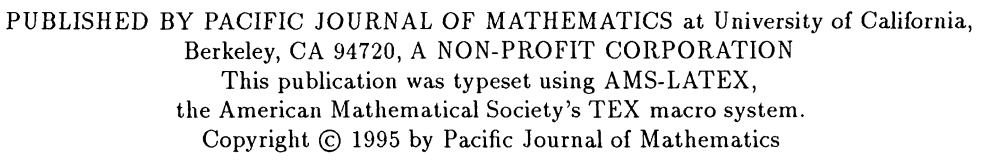




\section{PACIFIC JOURNAL OF MATHEMATICS}

\section{Volume $171 \quad$ No. $2 \quad$ December 1995}

On $H^{p}$-solutions of the Bezout equation

ERIC AMAR, JOAQUIM BRUNA FLORIS and ARTUR NiCOLAU

Amenable correspondences and approximation properties for von Neumann algebras

309

Claire ANANTHARAman-DElaroche

On moduli of instanton bundles on $\mathbb{P}^{2 n+1}$

VinCENZO ANCONA and Giorgio MaRia OtTAVIANI

Minimal surfaces with catenoid ends

JORGEN BERGLUND and WAYNE ROSSMAN

Permutation model for semi-circular systems and quantum random walks

PHILIPPE BIANE

The Neumann problem on Lipschitz domains in Hardy spaces of order less than one

RUSSELL M. BROWN

Matching theorems for twisted orbital integrals

409

REBECCA A. HERB

Uniform algebras generated by holomorphic and pluriharmonic functions on strictly

pseudoconvex domains

ALEXANDER IzZO

Quantum Weyl algebras and deformations of $U(g)$

NAIHUAN JING and JAMES ZHANG

Calcul du nombre de classes des corps de nombres

STÉPHANE LOUBOUTIN

On geometric properties of harmonic Lip ${ }_{1}$-capacity

PertTi MatTila and P. V. PARAmonov

Reproducing kernels and composition series for spaces of vector-valued holomorphic functions

Bent ØRsted and Genkai Zhang

Iterated loop modules and a filtration for vertex representation of toroidal Lie algebras

S. ESWARA RAO

The intrinsic mountain pass

MARTIN SCHECHTER

A Frobenius problem on the knot space

RON G. WANG

On complete metrics of nonnegative curvature on 2-plane bundles

DAVID YANG

Correction to: "Free Banach-Lie algebras, couniversal Banach-Lie groups, and more"

VLADIMIR G. PESTOV

Correction to: "Asymptotic radial symmetry for solutions of $\Delta u+e^{u}=0$ in a punctured disc"

KAI Seng (KAISing) CHOU (Tso) and Tom YAU-Heng WAN 